\title{
EXPONENTIAL QUADRATURE RULES WITHOUT ORDER REDUCTION FOR INTEGRATING LINEAR INITIAL BOUNDARY VALUE PROBLEMS*
}

\author{
BEGOÑA CANO $†$ AND MARÍA JESÚS MORETA $\ddagger$
} order reduction

\begin{abstract}
In this paper a technique is suggested to integrate linear initial boundary value problems with exponential quadrature rules in such a way that the order in time is as high as possible. A thorough error analysis is given for both the classical approach of integrating the problem firstly in space and then in time and of doing it in the reverse order in a suitable manner. Time-dependent boundary conditions are considered with both approaches and full discretization formulas are given to implement the methods once the quadrature nodes have been chosen for the time integration and a particular (although very general) scheme is selected for the space discretization. Numerical experiments are shown which corroborate that, for example, with the suggested technique, order $2 s$ is obtained when choosing the $s$ nodes of Gaussian quadrature rule.
\end{abstract}

Key words. Exponential quadrature rules, linear initial boundary value problems, avoiding AMS subject classifications. 65M12 65M20

1. Introduction. Due to the recent development and improvement of Krylov methods [7, 11], exponential quadrature rules have become a valuable tool to integrate linear initial value partial differential problems [9]. This is because of the fact that the linear and stiff part of the problem can be 'exactly' integrated in an efficient way through exponential operators. Moreover, when the source term is nontrivial, a variations-of-constants formula and the interpolation of that source term in several nodes leads to the appearance of some $\varphi_{j}$-operators, to which Krylov techniques can also be applied.

However, in the literature [9], these methods have always been applied and analysed either on the initial value problem or on the initial boundary value one with vanishing or periodic boundary conditions. More precisely, when the linear and stiff operator is the infinitesimal generator of a strongly continuous semigroup in a certain Banach space. In such a case, it has been proved [9] that the exponential quadrature rule converges with global order $s$ if $s$ is the number of nodes being used for the interpolation of the source term.

There are no results concerning specifically how to deal with these methods when integrating the most common non-vanishing and time-dependent boundary conditions case. The only related reference is that of Lawson quadrature rules $[3,4,10]$ which differ from these methods in the fact that, not only the source term is interpolated, but all the integrand which turns up in the variations-of-constants formula. In such a way, with the latter methods, $\left\{\varphi_{j}\right\}$-operators (with $j \geq 1$ ) do not turn up. Only exponential functions (those corresponding to $j=0$ ) are present. In [3] a thorough error analysis is given which studies the strong order reduction which turns up with Lawson methods even in the vanishing boundary conditions case unless some even

*This work was funded by Ministerio de Economía y Competitividad, Junta de Castilla y León and FEDER through projects MTM 2015- 66837-P and VA024P17.

${ }^{\dagger}$ IMUVA, Departamento de Matemática Aplicada, Universidad de Valladolid, Paseo de Belén 7, 47011 Valladolid, Spain, (bego@mac.uva.es).

${ }^{\ddagger}$ IMUVA, Departamento de Análisis Económico, Facultad de Ciencias Económicas y Empresariales, Universidad Complutense de Madrid, Campus de Somosaguas, Pozuelo de Alarcón, 28223 Madrid (mjesusmoreta@ccee.ucm.es). 
more artificial additional vanishing boundary conditions are satisfied. Nevertheless, in [4], a technique is suggested to avoid that order reduction under homogeneous boundary conditions and to even tackle the time-dependent boundary case without order reduction. Moreover, the analysis there also includes the error coming from the space discretization.

The aim of this paper is to generalize that technique to the most common quadrature rules which are used in the literature and which also use $\left\{\varphi_{j}\right\}$-operators. Besides, we also include the error coming from the space discretization not only when avoiding order reduction but also with the classical approach. We will see that, with the technique which is suggested in this paper, we manage to get the order of the classical quadrature interpolatory rule. This implies that, by choosing the $s$ nodes carefully, we can manage to get even order $2 s$ in time, while the classical approach just leads to order $s$. On the other hand, in comparison with Lawson methods, at least when the nodes $c_{i}$ are different and different from zero, less exponential-type functions of matrices applied over vectors are required now when avoiding order reduction. (Compare (21)-(26)-(27) here with formula (4.12) in [4].)

The paper is structured as follows. Section 2 gives some preliminaries on the abstract framework in Banach spaces which is used for the time integration of the problem, on the definition of the exponential quadrature rules and on the general hypotheses which are used for the abstract space discretization. Then, Section 3 describes and makes a thorough error analysis of the classical method of lines, which integrates the problem firstly in space and then in time. Both vanishing and nonvanishing boundary conditions are considered there and several different results are obtained depending on the specific accuracy of the quadrature rule and on whether a parabolic assumption is satisfied. After that, the technique which is suggested in the paper to improve the order of accuracy in time is well described in Section 4. It consists of discretizing firstly in time with suitable boundary conditions and then in space. Therefore, the analysis is firstly performed on the local error of the time semidiscretization and then on the local and global error of the full scheme (21),(26),(27). Finally, Section 5 shows some numerical results which corroborate the theoretical results of the previous sections.

2. Preliminaries. As in [4], we consider the linear abstract initial boundary value problem in the complex Banach space $X$

$$
\begin{aligned}
u^{\prime}(t) & =A u(t)+f(t), \quad 0 \leq t \leq T, \\
u(0) & =u_{0}, \\
\partial u(t) & =g(t),
\end{aligned}
$$

where $D(A)$ is a dense subspace of $X$ and $u_{0}, f, g$ and the linear operators $A: D(A) \rightarrow$ $X$ and $\partial: D(A) \rightarrow Y$ satisfy the assumptions in $[1,4,12]$ so that problem (1) is wellposed in $B V / L^{\infty}$ sense. Moreover, because of those hypotheses, for $A_{0}=\left.A\right|_{\operatorname{ker}(\partial)}$, the semigroup $e^{t A_{0}}$ decays exponentially when $t \rightarrow \infty$ and the operator $A_{0}$ is invertible, which make that $\left\{\varphi_{j}\left(t A_{0}\right)\right\}_{j=0}^{\infty}$ are bounded operators for $t>0$, where $\left\{\varphi_{j}\right\}$ are the standard functions which are used in exponential methods [9]:

$$
\varphi_{j}\left(t A_{0}\right)=\frac{1}{t^{j}} \int_{0}^{t} e^{(t-\tau) A_{0}} \frac{\tau^{j-1}}{(j-1) !} d \tau
$$

It is well-known that they can be calculated in a recursive way through the formula

$$
\text { (3) } \quad \varphi_{k+1}(z)=\frac{\varphi_{k}(z)-1 / k !}{z}, \quad z \neq 0, \quad \varphi_{k+1}(0)=\frac{1}{(k+1) !}, \quad \varphi_{0}(z)=e^{z},
$$


and that these functions are bounded on the complex plane when $\operatorname{Re}(z) \leq 0$.

We also assume that the solution of (1) satisfies that, for a natural number $p$,

$$
A^{l} u^{(j)} \in C([0, T], X), \quad l+j \leq p+1 .
$$

When $A$ is a differential space operator, this assumption implies that the time derivatives of the solution are regular in space, but without imposing any restriction of annihilation on the boundary. Because of Theorem 3.1 in [1], this assumption is satisfied when the data $u_{0}, f$ and $g$ are regular and satisfy certain natural compatibility conditions at the boundary. More precisely, when the following is satisfied:

(i) $u_{0} \in D\left(A^{p+1}\right), g \in C^{p+2}([0, T], Y), f \in C^{p+1}([0, T], X)$,

(ii) $f^{(i)}(t) \in D\left(A^{p-i}\right), \quad 0 \leq i \leq p, \quad 0 \leq t \leq T$,

(iii) $\partial A^{j} u_{0}=g^{(j)}(0)-\sum_{i=0}^{j-1} \partial A^{j-i-1} f^{(i)}(0), \quad 0 \leq j \leq p$.

Moreover, the crucial boundary values for the technique that we suggest $\left(\partial A^{j} u\right)$ can be calculated from the given data in this way

$$
\partial A^{j} u(t)=g^{(j)}(t)-\sum_{l=0}^{j-1} \partial A^{l} f^{(j-1-l)}(t), \quad 0 \leq j \leq p .
$$

We will center on exponential quadrature rules [9] to time integrate (1). When applied to a finite-dimensional linear problem like

$$
U^{\prime}(t)=B U(t)+F(t)
$$

where $B$ is a matrix, these rules correspond to interpolating $F$ in $s$ nodes $\left\{c_{i}\right\}_{i=1}^{s}$ in the integral in the equality

$$
U\left(t_{n+1}\right)=e^{k B} U\left(t_{n}\right)+k \int_{0}^{1} e^{k(1-\theta) B} F\left(t_{n}+\theta k\right) d \theta,
$$

which is satisfied by the solutions of (5) when $t_{n+1}=t_{n}+k$. This yields

$$
U^{n+1}=e^{k B} U^{n}+k \sum_{i=1}^{s} b_{i}(k B) F\left(t_{n}+c_{i} k\right),
$$

with weights

$$
b_{i}(k B)=\int_{0}^{1} e^{k(1-\theta) B} l_{i}(\theta) d \theta,
$$

where $l_{i}$ are the Lagrange interpolation polynomials corresponding to the nodes $\left\{c_{i}\right\}_{i=1}^{s}$. We will define the values $\left\{a_{i j}\right\}_{i, j=1}^{s}$ in such a way that

$$
l_{i}(\theta)=a_{i, 1}+a_{i, 2} \theta+a_{i, 3} \frac{\theta^{2}}{2}+\cdots+a_{i, s} \frac{\theta^{s-1}}{(s-1) !} .
$$

From this,

$$
b_{i}(k B)=\int_{0}^{1} e^{k(1-\theta) B} l_{i}(\theta) d \theta=\frac{1}{k} \int_{0}^{k} e^{(k-\sigma) B} l_{i}\left(\frac{\sigma}{k}\right) d \sigma=\sum_{j=1}^{s} a_{i, j} \varphi_{j}(k B),
$$


for the functions $\varphi_{j}$ in (2), and the final formula for the integration of (5) is

$$
U^{n+1}=e^{k B} U^{n}+k \sum_{i, j=1}^{s} a_{i, j} \varphi_{j}(k B) F\left(t_{n}+c_{i} k\right) .
$$

We will consider an abstract spatial discretization which satisfies the same hypotheses as in [4] (Section 4.1) and which includes a big range of techniques. In such a way, for each parameter $h$ in a sequence $\left\{h_{j}\right\}_{j=1}^{\infty}$ such that $h_{j} \rightarrow 0, X_{h} \subset X$ is a finite dimensional space which approximates $X$ when $h_{j} \rightarrow 0$ and the elements in $D\left(A_{0}\right)$ are approximated in a subspace $X_{h, 0}$. The norm in $X_{h}$ is denoted by $\|\cdot\|_{h}$. The operator $A$ is then approximated by $A_{h}, A_{0}$ by $A_{h, 0}$ and the solution of the elliptic problem

$$
A w=F, \quad \partial w=g,
$$

is approximated by $R_{h} w+Q_{h} g$, where $R_{h} w \in X_{h, 0}$ is called the elliptic projection, $Q_{h} g \in X_{h}$ discretizes the boundary values and the following is satisfied:

$$
A_{h, 0} R_{h} w+A_{h} Q_{h} g=L_{h} F,
$$

for a projection operator $L_{h}: X \rightarrow X_{h, 0}$. We will also use $P_{h}=L_{h}-L_{h} Q_{h} \partial$ and we remind part of hypothesis (H3) in [4], which states that, for a subspace $Z$ of $X$ with norm $\|\cdot\|_{Z}$, whenever $u \in Z$,

$$
\left\|A_{h, 0}\left(R_{h}-P_{h}\right) u\right\|_{h} \leq \varepsilon_{h}\|u\|_{Z},
$$

for $\varepsilon_{h}$ decreasing with $h$ and, therefore, this gives a bound for the error in the space discretization of operator $A$.

Moreover, we will assume that this additional hypothesis is satisfied:

(HS) $\left\|A_{h, 0}^{-1} A_{h} Q_{h}\right\|_{h}$ is bounded independently of $h$ for small enough $h$. Considering (9), this in fact corresponds to a discrete maximum principle, which would be simulating the continuous maximum principle which is satisfied because of one of the hypotheses in [4].

3. Classical approach: Discretizing firstly in space and then in time. When considering vanishing boundary conditions in (1) (which has been classically done in the literature with exponential methods [9]), discretizing first in space and then in time leads to the following semidiscrete problem in $X_{h, 0}$ :

$$
\begin{aligned}
U_{h}^{\prime}(t) & =A_{h, 0} U_{h}(t)+L_{h} f(t), \\
U_{h}(0) & =L_{h} u(0) .
\end{aligned}
$$

When integrating this problem with an exponential quadrature rule which is based on $s$ nodes (8), the following scheme arises:

$$
U_{h}^{n+1}=e^{k A_{h, 0}} U_{h}^{n}+k \sum_{i, j=1}^{s} a_{i, j} \varphi_{j}\left(k A_{h, 0}\right) L_{h} f\left(t_{n}+c_{i} k\right) .
$$

Denoting by $\rho_{h, n+1}$ to $U_{h}\left(t_{n+1}\right)-\bar{U}_{h}^{n+1}$ where $\bar{U}_{h}^{n+1}$ is the result of applying (11) from $U_{h}\left(t_{n}\right)$ instead of $U_{h}^{n}$; and $e_{h, n+1}$ to $U_{h}\left(t_{n+1}\right)-U_{h}^{n+1}$ the following result follows:

Theorem 1. Whenever $g(t)=0$ in $(1), u \in C([0, T], Z)$ and $f \in C^{s}([0, T], X)$,

(i) $\rho_{h, n}=O\left(k^{s+1}\right)$,

(ii) $e_{h, n}=O\left(k^{s}\right)$, 
(iii) $L_{h} u\left(t_{n}\right)-U_{h}^{n}=O\left(k^{s}+\varepsilon_{h}\right)$, where the constants in Landau notation are independent of $k$ and $h$.

Proof. (i) comes from the fact that the difference between $f\left(t_{n}+k \tau\right)$ and its interpolant $I\left(f\left(t_{n}+k \tau\right)\right)$ in those nodes is $O\left(k^{s}\right)$. More explicitly, by using (6) and the definition of $\bar{U}_{h}^{n+1}$,

$$
\rho_{h, n+1}=U_{h}\left(t_{n+1}\right)-\bar{U}_{h}^{n+1}=k \int_{0}^{1} e^{k(1-\theta) A_{h, 0}} L_{h}\left[f\left(t_{n}+k \theta\right)-I\left(f\left(t_{n}+k \theta\right)\right)\right] d \theta .
$$

Now, taking into account hypotheses (H1)-(H2) in [4], $e^{k(1-\tau) A_{h, 0}}$ and $L_{h}$ are bounded with $h$, and the result follows.

Then, (ii) is deduced from the classical argument for the global error once the local error is bounded. Finally, (iii) comes from (ii) and the decomposition

$$
L_{h} u\left(t_{n}\right)-U_{h}^{n}=\left[L_{h} u\left(t_{n}\right)-U_{h}\left(t_{n}\right)\right]+\left[U_{h}\left(t_{n}\right)-U_{h}^{n}\right],
$$

by noticing that, for the first term, as $g=0$, it happens that

$L_{h} \dot{u}(t)-\dot{U}_{h}(t)=A_{h, 0}\left(R_{h} u(t)-U_{h}(t)\right)=A_{h, 0}\left(L_{h} u(t)-U_{h}(t)\right)+A_{h, 0}\left(R_{h} u(t)-P_{h} u(t)\right)$, $L_{h} u(0)-U_{h}(0)=0$.

Then, because of (10), $L_{h} u(t)-U_{h}(t)=\int_{0}^{t} e^{(t-s) A_{h, 0}} O\left(\varepsilon_{h}\right) d s=O\left(\varepsilon_{h}\right)$.

We also have this finer result, which implies global order $s+1$ under more restrictive hypotheses.

THEOREM 2. Let us assume that $g(t)=0$ in (1), u belongs to $C([0, T], Z), f$ to $C^{s+2}([0, T], X)$, the interpolatory quadrature rule which is based on $\left\{c_{i}\right\}_{i=1}^{s}$ integrates exactly polynomials of degree less than or equal to $s$ and this bound holds

$$
\left\|k A_{h, 0} \sum_{r=1}^{n-1} e^{r k A_{h, 0}}\right\|_{h} \leq C, \quad 0 \leq n k \leq T .
$$

Then,

(i) $A_{h, 0}^{-1} \rho_{h, n}=O\left(k^{s+2}\right)$,

(ii) $e_{h, n}=O\left(k^{s+1}\right)$,

(iii) $L_{h} u\left(t_{n}\right)-U_{h}^{n}=O\left(k^{s+1}+\varepsilon_{h}\right)$,

where the constants in Landau notation are independent of $k$ and $h$.

Proof. To prove (i), it suffices to consider the following formula for the interpolation error which is valid when $f \in C^{s+1}$ :

$$
f\left(t_{n}+k \theta\right)-I\left(f\left(t_{n}+k \theta\right)\right)=k^{s}\left[f^{(s)}\left(t_{n}\right) \prod_{i=1}^{s}\left(\theta-c_{i}\right)+O(k)\right] .
$$


Then, substituting in (12) and multiplying by $A_{h, 0}^{-1}$,

$$
\begin{aligned}
A_{h, 0}^{-1} \rho_{h, n+1}= & k^{s+2} \int_{0}^{1}(1-\theta)\left(k(1-\theta) A_{h, 0}\right)^{-1}\left[e^{k(1-\theta) A_{h, 0}}-I\right] L_{h}\left[f^{(s)}\left(t_{n}\right) \prod_{i=1}^{s}\left(\theta-c_{i}\right)+O(k)\right] d \theta \\
& +k^{s+2} \int_{0}^{1} k^{-1} A_{h, 0}^{-1} L_{h}\left[f^{(s)}\left(t_{n}\right) \prod_{i=1}^{s}\left(\theta-c_{i}\right)+O(k)\right] d \theta \\
= & k^{s+2} \int_{0}^{1}(1-\theta) \varphi_{1}\left(k(1-\theta) A_{h, 0}\right) L_{h} f^{(s)}\left(t_{n}\right) \prod_{i=1}^{s}\left(\theta-c_{i}\right) d \theta \\
& +k^{s+1}\left(\int_{0}^{1} \prod_{i=1}^{s}\left(\theta-c_{i}\right) d \theta\right) A_{h, 0}^{-1} L_{h} f^{(s)}\left(t_{n}\right)+O\left(k^{s+2}\right)=O\left(k^{s+2}\right),
\end{aligned}
$$

where we have used (3), (H1)-(H2) in [4] and the fact that the integral in brackets vanishes because the interpolatory quadrature rule is exact for polynomials of degree $s$.

As for (ii), a summation-by-parts argument like that given in [5] for splitting exponential methods also applies here because of hypothesis (13) and the fact that $f \in C^{s+2}$. Finally, (iii) follows in the same way as in the proof of Theorem 1.

REMARK 3. Notice that, when $\|\cdot\|_{h}$ is the discrete $L^{2}$-norm associated with the rectangular rule over some uniformly distributed nodal values, $\left\|k A_{h, 0} \sum_{r=1}^{n-1} e^{r k A_{h, 0}}\right\|_{h}$ coincides with the Euclidean norm of the associated matrix. Therefore, when $A_{h, 0}$ is represented by a symmetric matrix, this norm also coincides with its spectral radius. As, for each eigenvalue $\lambda_{h}$,

$$
\left|k \lambda_{h} \sum_{r=1}^{n-1} e^{r k \lambda_{h}}\right|=k\left|\lambda_{h}\right| \frac{e^{k \lambda_{h}}-e^{t_{n} \lambda_{h}}}{1-e^{k \lambda_{h}}},
$$

if the eigenvalues of the matrix which represents $A_{h, 0}$ are negative, the latter norm is uniformly bounded in the negative real axis, and therefore (13) follows. In fact, this bound has been proved in [8] for analytic semigroups covering the case in which (1) corresponds to parabolic problems. Therefore it seems natural that it is also satisfied by a suitable space discretization of them.

On the other hand, when $g \not \equiv 0$ in (1), the semidiscretized problem which arises is

$$
\begin{aligned}
U_{h}^{\prime}(t) & =A_{h, 0} U_{h}(t)+A_{h} Q_{h} g(t)+L_{h} Q_{h}\left(\partial f(t)-g^{\prime}(t)\right)+P_{h} f(t), \\
U_{h}(0) & =P_{h} u(0) .
\end{aligned}
$$

In a similar way as before, the local error would be given by

$$
\begin{aligned}
k \int_{0}^{1} e^{k(1-\theta) A_{h, 0}} & {\left[A_{h} Q_{h}\left[g\left(t_{n}+k \theta\right)-I\left(g\left(t_{n}+k \theta\right)\right)\right]\right.} \\
& +L_{h} Q_{h}\left[\partial f\left(t_{n}+k \theta\right)-g^{\prime}\left(t_{n}+k \theta\right)-I\left(\partial f\left(t_{n}+k \theta\right)-g^{\prime}\left(t_{n}+k \theta\right)\right)\right. \\
& \left.+P_{h}\left[f\left(t_{n}+k \theta\right)-I\left(f\left(t_{n}+k \theta\right)\right)\right]\right] d \theta .
\end{aligned}
$$

Again, when $g \in C^{s+1}([0, T], Y)$ and $f \in C^{s}([0, T], X)$, the error of interpolation will be $O\left(k^{s}\right)$. However, although $L_{h} Q_{h}$ and $P_{h}$ are bounded [4], $A_{h} Q_{h}$ is not bounded any more. That is why we state the following result which bounds in fact $A_{h, 0}^{-1} \rho_{h, n}$ by using (HS) and which proof for the global error is the same as in Theorem 2 . 
TheOREM 4. Let us assume that $g(t) \not \equiv 0$ in (1), $u$ belongs to $C([0, T], Z), g$ to $C^{s+2}([0, T], Y), f$ to $C^{s+1}([0, T], X)$, and the bound (13) holds. Then,

(i) $A_{h, 0}^{-1} \rho_{h, n}=O\left(k^{s+1}\right)$,

(ii) $e_{h, n}=O\left(k^{s}\right)$,

(iii) $L_{h} u\left(t_{n}\right)-U_{h}^{n}=O\left(k^{s}+\varepsilon_{h}\right)$, where the constants in Landau notation are independent of $k$ and $h$.

REMARK 5. As in Remark 3, if $A_{h, 0}$ is is represented by a symmetric matrix with negative eigenvalues and the discrete $L^{2}$-norm associated with the rectangular rule is considered, $\| k A_{h, 0} e^{k(1-\theta) A_{h, 0} \|_{h}}$ coincides with its spectral radius. As for each eigenvalue $\lambda_{h}$ of $A_{h, 0}$,

$$
\int_{0}^{1} k\left|\lambda_{h}\right| e^{k(1-\theta) \lambda_{h}} d \theta=\int_{0}^{1} \frac{d}{d \theta}\left(e^{k(1-\theta) \lambda_{h}}\right) d \theta=1-e^{k \lambda_{h}} \leq 1,
$$

considering this in the first part of (14) explains that the local error $\rho_{h, n}$ behaves as $O\left(k^{s}\right)$ under the rest of hypotheses of Theorem 4.

In any case, we want to remark in this section that accuracy has been lost with respect to the vanishing boundary conditions case since order reduction turns up at least for the local error and, in many cases, also for the global error.

4. Suggested approach: Discretizing firstly in time and then in space. In this section, we directly tackle the nonvanishing boundary conditions case by discretizing in a suitable way firstly in time and then in space. We will see that we manage to get at least the same order as with the classical approach when vanishing boundary conditions are present, but even a much higher order some times.

Let us suggest how to apply the exponential quadrature rule (8) directly to (1). When $g=0, B$ in (8) is directly substituted by $A_{0}$ and there is no problem because $e^{k A_{0}}$ and $\varphi_{j}\left(k A_{0}\right)$ have perfect sense over $X$. However, it has no sense to do that when $g \neq 0$ because $A$ is not $A_{0}$ any more. For Lawson methods, for which just exponential functions appear, instead of $e^{\tau A_{0}} \alpha$, it was suggested in [4] to consider $v_{0}(\tau)$ as the solution of

$$
\begin{aligned}
v_{0}^{\prime}(\tau) & =A v_{0}(\tau), \\
v_{0}(0) & =\alpha \\
\partial v_{0}(\tau) & =\sum_{l=0}^{p} \frac{\tau^{l}}{l !} \partial A^{l} \alpha,
\end{aligned}
$$

whenever $\alpha \in D\left(A^{p}\right)$. In such a way, if $\alpha \in D\left(A^{p+1}\right)$,

$$
v_{0}(\tau)=\sum_{l=0}^{p} \frac{\tau^{l}}{l !} A^{l} \alpha+\tau^{p+1} \varphi_{p+1}\left(\tau A_{0}\right) A^{p+1} \alpha,
$$

which resembles the formal analytic expansion of the exponential of $\tau A$ applied over $\alpha$. In this manuscript then, whenever $\alpha \in D\left(A^{p}\right)$, for $j=1, \ldots, s$, instead of $\varphi_{j}\left(\tau A_{0}\right) \alpha$, we suggest to consider the following functions :

$$
v_{j}(\tau)=\sum_{l=0}^{p-1} \frac{\tau^{l}}{(l+j) !} A^{l} \alpha+\tau^{p} \varphi_{p+j}\left(\tau A_{0}\right) A^{p} \alpha .
$$

This resembles the formal analytic expansion of $\varphi_{j}$ when evaluated at $\tau A$ and applied over $\alpha$. (Notice that, for $j=0$, this would correspond to (16) changing $p$ by $p-1$. As the functions $\varphi_{j}$ are multiplied by $k$ in (8), we need one less term in this expansion.) 
Therefore, imitating (8), we suggest to consider as continuous numerical approximation $u_{n+1}$ from the previous $u_{n}$,

$$
\begin{aligned}
u_{n+1}= & \tilde{v}_{0, n, t_{n}}(k) \\
& +k \sum_{i, j=1}^{s} a_{i, j}\left[\sum_{l=0}^{p-1} \frac{k^{l}}{(l+j) !} A^{l} f\left(t_{n}+c_{i} k\right)+k^{p} \varphi_{p+j}\left(k A_{0}\right) A^{p} f\left(t_{n}+c_{i} k\right)\right],
\end{aligned}
$$

where $\tilde{v}_{0, n, t_{n}}(\tau)$ is the generalised solution of

$$
\begin{aligned}
\tilde{v}_{0, n, t_{n}}^{\prime}(\tau) & =A \tilde{v}_{0, n, t_{n}}(\tau), \\
\tilde{v}_{0, n, t_{n}}(0) & =u_{n}, \\
\partial \tilde{v}_{0, n, t_{n}}(\tau) & =\sum_{l=0}^{p} \frac{\tau^{l}}{l !} \partial A^{l} u\left(t_{n}\right) .
\end{aligned}
$$

We notice that $\tilde{v}_{0, n, t_{n}}(\tau)$ is the same type of function which was considered with Lawson methods. In case $u_{n}$ were $u\left(t_{n}\right)$, it corresponds to (15) with $\alpha=u\left(t_{n}\right)$ and therefore $\tilde{v}_{0, n, t_{n}}(k)$ is the same as (16) with $\tau=k$ and $\alpha=u\left(t_{n}\right)$. If just $\partial u\left(t_{n}\right)=\partial u_{n}$, that solution would be the one given in Lemma 3.1 of [4] and, even in the case that $\partial u_{n} \neq \partial u\left(t_{n}\right), \tilde{v}_{0, n, t_{n}}(\tau)$ would be understood in the sense described in Remark 2.3 of [4]. However, because of the assumed regularity of $f$ we want to remark here that we do not need an initial boundary value problem similar to (15) to define the rest of terms in (18). Nevertheless, we seek a differential equation which the functions in (17) satisfy so that it is not necessary to calculate $A^{l} f\left(t_{n}+c_{i} k\right)(l=0, \ldots, p)$ on the whole domain. For that, let us first consider the following lemma.

Lemma 6. For $j \geq 1$ and $\alpha \in X$,

$$
\frac{d}{d \tau} \varphi_{j}\left(\tau A_{0}\right) \alpha=\left(A_{0}-\frac{j}{\tau} I\right) \varphi_{j}\left(\tau A_{0}\right) \alpha+\frac{1}{(j-1) ! \tau} \alpha, \quad \tau>0
$$

where, for $\alpha \in X \backslash D\left(A_{0}\right)$, as $D\left(A_{0}\right)$ is dense in $X, A_{0} \varphi_{j}\left(\tau A_{0}\right) \alpha$ is understood as the corresponding limit of a sequence in $D\left(A_{0}\right)$. (This limit exists because, over $D\left(A_{0}\right)$, $A_{0} \varphi_{j}\left(\tau A_{0}\right)=\left[\varphi_{j-1}\left(\tau A_{0}\right)-\frac{1}{(j-1) !} I\right] / \tau$, which is a bounded operator according to the assumed hypotheses in the preliminaries.)

Proof. Assuming firstly that $\alpha \in D\left(A_{0}\right)$ and considering (2),

$$
\begin{aligned}
& \frac{d}{d \tau}\left[\frac{1}{\tau^{j}} \int_{0}^{\tau} e^{(\tau-\theta) A_{0}} \alpha \frac{\theta^{j-1}}{(j-1) !} d \theta\right] \\
& =-\frac{j}{\tau^{j+1}} \int_{0}^{\tau} e^{(\tau-\theta) A_{0}} \alpha \frac{\theta^{j-1}}{(j-1) !} d \theta+\frac{1}{\tau^{j}} \frac{\tau^{j-1}}{(j-1) !} \alpha+\frac{1}{\tau^{j}} \int_{0}^{\tau} A_{0} e^{(\tau-\theta) A_{0}} \alpha \frac{\theta^{j-1}}{(j-1) !} d \theta \\
& =\left(A_{0}-\frac{j}{\tau} I\right) \varphi_{j}\left(\tau A_{0}\right) \alpha+\frac{1}{(j-1) ! \tau} \alpha .
\end{aligned}
$$

The result on the whole space $X$ comes from density.

From here, the next result follows:

LEMMA 7. The function $v_{j}(\tau)$ in (17) satisfies the following initial boundary value 
problem:

$$
\begin{aligned}
v_{j}^{\prime}(\tau) & =\left(A-\frac{j}{\tau}\right) v_{j}(\tau)+\frac{1}{(j-1) ! \tau} \alpha, \quad \tau>0, \\
v_{j}(0) & =\frac{1}{j !} \alpha, \\
\partial v_{j}(\tau) & =\sum_{l=0}^{p-1} \frac{\tau^{l}}{(l+j) !} \partial A^{l} \alpha .
\end{aligned}
$$

Proof. Notice that, using Lemma 6,

$$
\begin{aligned}
v_{j}^{\prime}(\tau)= & \sum_{l=1}^{p-1} \frac{l \tau^{l-1}}{(l+j) !} A^{l} \alpha+p \tau^{p-1} \varphi_{p+j}\left(\tau A_{0}\right) A^{p} \alpha \\
& +\tau^{p}\left[\left(A_{0}-\frac{p+j}{\tau} I\right) \varphi_{p+j}\left(\tau A_{0}\right)+\frac{1}{(p+j-1) ! \tau} I\right] A^{p} \alpha \\
= & \sum_{l=1}^{p-1} \frac{l \tau^{l-1}}{(l+j) !} A^{l} \alpha+\frac{\tau^{p-1}}{(p+j-1) !} A^{p} \alpha-j \tau^{p-1} \varphi_{p+j}\left(\tau A_{0}\right) A^{p} \alpha+\tau^{p} A_{0} \varphi_{p+j}\left(\tau A_{0}\right) A^{p} \alpha
\end{aligned}
$$

On the other hand,

$$
\begin{aligned}
& \left(A-\frac{j}{\tau}\right) v_{j}(\tau)+\frac{1}{(j-1) ! \tau} \alpha \\
& =\sum_{l=0}^{p-1} \frac{\tau^{l}}{(l+j) !} A^{l+1} \alpha+\tau^{p} A \varphi_{p+j}\left(\tau A_{0}\right) A^{p} \alpha-j \sum_{l=1}^{p-1} \frac{\tau^{l-1}}{(l+j) !} A^{l} \alpha-j \tau^{p-1} \varphi_{p+j}\left(\tau A_{0}\right) A^{p} \alpha \\
& =\sum_{m=1}^{p-1} \frac{m}{(m+j) !} \tau^{m-1} A^{m} \alpha+\frac{\tau^{p-1}}{(p-1+j) !} A^{p} \alpha-j \tau^{p-1} \varphi_{p+j}\left(\tau A_{0}\right) A^{p} \alpha+\tau^{p} A \varphi_{p+j}\left(\tau A_{0}\right) A^{p} \alpha
\end{aligned}
$$

where the change $m=l+1$ has been used in the third line for the first sum. Therefore, the lemma is proved taking also into account that $\varphi_{p+j}\left(\tau A_{0}\right) A^{p} \alpha \in D\left(A_{0}\right)$.

With Lawson methods [4], starting from a previous approximation $U_{h, n}$ to $P_{h} u\left(t_{n}\right)$ and discretizing (19) in space led to a term like

$$
\begin{aligned}
V_{h, n, 0}(k)= & e^{k A_{h, 0}} U_{h, n}+\sum_{j=1}^{p} k^{j} \varphi_{j}\left(k A_{h, 0}\right)\left[A_{h} Q_{h} \partial A^{j-1} u\left(t_{n}\right)-L_{h} Q_{h} \partial A^{j} u\left(t_{n}\right)\right] \\
& +k^{p+1} \varphi_{p+1}\left(k A_{h, 0}\right) A_{h} Q_{h} \partial A^{p} u\left(t_{n}\right),
\end{aligned}
$$

which is the approximation which corresponds to the first term in (8).

For the rest of the terms in (8), we suggest to discretize (20) in space with $\alpha=$ $f\left(t_{n}+c_{i} k\right)$. In such a way, the following system turns up:

$$
\begin{aligned}
V_{h, j, n, i}^{\prime}(\tau)+L_{h} Q_{h} \partial \hat{v}_{j, n, i}^{\prime}(\tau)= & A_{h, 0} V_{h, j, n, i}(\tau)+A_{h} Q_{h} \partial \hat{v}_{j, n, i}(\tau)-\frac{j}{\tau}\left[V_{h, j, n, i}(\tau)+L_{h} Q_{h} \partial \hat{v}_{j, n, i}(\tau)\right] \\
& +\frac{1}{(j-1) ! \tau}\left[P_{h} f\left(t_{n}+c_{i} k\right)+L_{h} Q_{h} \partial f\left(t_{n}+c_{i} k\right)\right], \\
V_{h, j, n, i}(0)+L_{h} Q_{h} \partial \hat{v}_{j, n, i}(0)= & \frac{1}{j !} L_{h} f\left(t_{n}+c_{i} k\right),
\end{aligned}
$$


where

$$
\hat{v}_{j, n, i}(\tau)=\sum_{l=0}^{p-1} \frac{\tau^{l}}{(l+j) !} A^{l} f\left(t_{n}+c_{i} k\right) .
$$

This can be rewritten as

$$
\begin{aligned}
V_{h, j, n, i}^{\prime}(\tau)= & \left(A_{h, 0}-\frac{j}{\tau} I\right) V_{h, j, n, i}(\tau)+A_{h} Q_{h} \partial \hat{v}_{j, n, i}(\tau)+\frac{1}{(j-1) ! \tau} P_{h} f\left(t_{n}+c_{i} k\right) \\
& +L_{h} Q_{h} \partial\left[\frac{1}{(j-1) ! \tau} f\left(t_{n}+c_{i} k\right)-\frac{j}{\tau} \hat{v}_{j, n, i}(\tau)-\hat{v}_{j, n, i}^{\prime}(\tau)\right], \\
(22) V_{h, j, n, i}(0)= & \frac{1}{j !} P_{h} f\left(t_{n}+c_{i} k\right) .
\end{aligned}
$$

With the same arguments as in Lemma $6, \varphi_{j}\left(\tau A_{h, 0}\right) P_{h} f\left(t_{n}+c_{i} k\right)$ is the solution of

$$
\begin{aligned}
& W_{h, j, n, i}^{\prime}(\tau)=\left(A_{h, 0}-\frac{j}{\tau} I\right) W_{h, j}(\tau)+\frac{1}{(j-1) ! \tau} P_{h} f\left(t_{n}+c_{i} k\right), \\
& W_{h, j, n, i}(0)=\frac{1}{j !} P_{h} f\left(t_{n}+c_{i} k\right) .
\end{aligned}
$$

Therefore, in order to solve (22), we are interested in finding

$$
Z_{h, j, n, i}(\tau)=V_{h, j, n, i}(\tau)-W_{h, j, n, i}(\tau),
$$

which is the solution of

$$
\begin{aligned}
Z_{h, j, n, i}^{\prime}(\tau)= & \left(A_{h, 0}-\frac{j}{\tau} I\right) Z_{h, j, n, i}(\tau)+A_{h} Q_{h} \partial \hat{v}_{j, n, i}(\tau) \\
& +L_{h} Q_{h} \partial\left[\frac{1}{(j-1) ! \tau} f\left(t_{n}+c_{i} k\right)-\frac{j}{\tau} \hat{v}_{j, n, i}(\tau)-\hat{v}_{j, n, i}^{\prime}(\tau)\right], \\
Z_{h, j, n, i}(0)= & 0 .
\end{aligned}
$$

Now, using the first line of (20) for the boundary with $\alpha=f\left(t_{n}+c_{i} k\right)$,

$$
\begin{aligned}
& \partial\left[\frac{1}{(j-1) ! \tau} f\left(t_{n}+c_{i} k\right)-\frac{j}{\tau} \hat{v}_{j, n, i}(\tau)-\hat{v}_{j, n, i}^{\prime}(\tau)\right]=-\partial A v_{j, n, i}(\tau) \\
& =-\sum_{l=0}^{p-1} \frac{\tau^{l}}{(l+j) !} \partial A^{l+1} f\left(t_{n}+c_{i} k\right)-\tau^{p} \partial A_{0} \varphi_{p+j}\left(\tau A_{0}\right) A^{p} f\left(t_{n}+c_{i} k\right),
\end{aligned}
$$

the fact that

$\tau^{p} A_{0} \frac{1}{\tau^{p+j}} \int_{0}^{\tau} e^{(\tau-\sigma) A_{0}} \frac{\sigma^{p+j-1}}{(p+j-1) !} A^{p} f\left(t_{n}+c_{i} k\right) d \sigma$

$=-\left.\frac{1}{\tau^{j}} e^{(\tau-\sigma) A_{0}} \frac{\sigma^{p+j-1}}{(p+j-1) !}\right|_{\sigma=0} ^{\sigma=\tau} A^{p} f\left(t_{n}+c_{i} k\right)+\frac{1}{\tau^{j}} \int_{0}^{\tau} e^{(\tau-\sigma) A_{0}} \frac{\sigma^{p+j-2}}{(p+j-2) !} A^{p} f\left(t_{n}+c_{i} k\right) d \sigma$

$=-\frac{\tau^{p-1}}{(p+j-1) !} A^{p} f\left(t_{n}+c_{i} k\right)+\tau^{p-1} \varphi_{p+j-1}\left(\tau A_{0}\right) A^{p} f\left(t_{n}+c_{i} k\right)$,

and that the boundary of the second term vanishes, it follows that

$$
\partial\left[\frac{1}{(j-1) ! \tau} f\left(t_{n}+c_{i} k\right)-\frac{j}{\tau} \hat{v}_{j, n, i}(\tau)-\hat{v}_{j, n, i}^{\prime}(\tau)\right]=-\sum_{l=0}^{p-2} \frac{\tau^{l}}{(l+j) !} \partial A^{l+1} f\left(t_{n}+c_{i} k\right) .
$$


Using this in (24),

$$
\begin{aligned}
& Z_{h, j, n, i}(\tau) \\
& =\int_{0}^{\tau} e^{\int_{\theta}^{\tau}\left(A_{h, 0}-\frac{j}{\sigma} I\right) d \sigma} \sum_{l=0}^{p-2} \frac{\theta^{l}}{(l+j) !}\left[A_{h} Q_{h} \partial A^{l} f\left(t_{n}+c_{i} k\right)-L_{h} Q_{h} \partial A^{l+1} f\left(t_{n}+c_{i} k\right)\right. \\
& \left.+\frac{\theta^{p-1}}{(p-1+j) !} A_{h} Q_{h} \partial A^{p-1} f\left(t_{n}+c_{i} k\right)\right] d \theta \\
& =\sum_{l=0}^{p-2} \int_{0}^{\tau} e^{A_{h, 0}(\tau-\theta)} \frac{\theta^{j+l}}{\tau^{j}(l+j) !}\left[A_{h} Q_{h} \partial A^{l} f\left(t_{n}+c_{i} k\right)-L_{h} Q_{h} \partial A^{l+1} f\left(t_{n}+c_{i} k\right)\right] d \theta \\
& +\int_{0}^{\tau} e^{A_{h, 0}(\tau-\theta)} \frac{\theta^{p-1+j}}{\tau^{j}(p-1+j) !} A_{h} Q_{h} \partial A^{p-1} f\left(t_{n}+c_{i} k\right) d \theta \\
& =\sum_{l=0}^{p-2} \tau^{l+1} \varphi_{j+l+1}\left(\tau A_{h, 0}\right)\left[A_{h} Q_{h} \partial A^{l} f\left(t_{n}+c_{i} k\right)-L_{h} Q_{h} \partial A^{l+1} f\left(t_{n}+c_{i} k\right)\right] \\
& (25)+\tau^{p} \varphi_{p+j}\left(\tau A_{h, 0}\right) A_{h} Q_{h} \partial A^{p-1} f\left(t_{n}+c_{i} k\right) .
\end{aligned}
$$

Therefore, using (23),

$V_{h, j, n, i}(k)=\varphi_{j}\left(k A_{h, 0}\right) P_{h} f\left(t_{n}+c_{i} k\right)$

$$
+\sum_{l=0}^{p-2} k^{l+1} \varphi_{j+l+1}\left(k A_{h, 0}\right)\left[A_{h} Q_{h} \partial A^{l} f\left(t_{n}+c_{i} k\right)-L_{h} Q_{h} \partial A^{l+1} f\left(t_{n}+c_{i} k\right)\right],
$$

(26) $+k^{p} \varphi_{p+j}\left(k A_{h, 0}\right) A_{h} Q_{h} \partial A^{p-1} f\left(t_{n}+c_{i} k\right)$,

and the overall exponential quadrature rule would be given by

$$
\begin{aligned}
U_{h, 0} & =P_{h} u_{0}, \\
U_{h, n+1} & =V_{h, n, 0}(k)+k \sum_{i, j=1}^{s} a_{i, j} V_{h, j, n, i}(k),
\end{aligned}
$$

with $V_{h, n, 0}(k)$ in $(21)$ and $V_{h, j, n, i}(k)$ in $(26)$.

4.1. Time semidiscretization error. Let us first study just the error after time discretization. The local truncation error is well-known to be given by $\rho_{n}=$ $u\left(t_{n+1}\right)-\bar{u}_{n+1}$, where $\bar{u}_{n+1}$ is given by expression (18) substituting $u_{n}$ by $u\left(t_{n}\right)$.

Let us first consider the following general result, which will allow to conclude more particular results depending on the choice of the values $\left\{c_{i}\right\}_{i=1}^{s}$.

LEMma 8. Under the assumptions of regularity (4), the local truncation error satisfies

$\rho_{n}=\sum_{m=1}^{p} k^{m}\left[\sum_{r=0}^{m-1}\left(\frac{1}{m !}-\frac{1}{r !} \sum_{l=1}^{s} \frac{1}{(m-r-1+l) !} \sum_{i=1}^{s} c_{i}^{r} a_{i l}\right) A^{m-r-1} f^{(r)}\left(t_{n}\right)\right]+O\left(k^{p+1}\right)$. 
Proof. Notice that $\bar{u}_{n+1}$ can be written as

$$
\begin{aligned}
\bar{u}_{n+1} & =u\left(t_{n}\right)+\sum_{j=1}^{p} k^{j}\left[\frac{1}{j !} A^{j} u\left(t_{n}\right)+\sum_{i, l=1}^{s} a_{i, l} \frac{1}{(j-1+l) !} A^{j-1} f\left(t_{n}+c_{i} k\right)\right]+O\left(k^{p+1}\right) \\
& =u\left(t_{n}\right)+\sum_{j=1}^{p} k^{j}\left[\frac{1}{j !} A^{j} u\left(t_{n}\right)+\sum_{i, l=1}^{s} a_{i, l} \frac{1}{(j-1+l) !} \sum_{r=0}^{p-j} \frac{c_{i}^{r} k^{r}}{r !} A^{j-1} f^{(r)}\left(t_{n}\right)\right]+O\left(k^{p+1}\right) \\
& =u\left(t_{n}\right)+\sum_{m=1}^{p} k^{m}\left[\frac{1}{m !} A^{m} u\left(t_{n}\right)+\sum_{i, l=1}^{s} a_{i, l} \sum_{r=0}^{m-1} \frac{c_{i}^{r}}{(m-r-1+l) ! r !} A^{m-r-1} f^{(r)}\left(t_{n}\right)\right]+O\left(k^{p+1}\right),
\end{aligned}
$$

where the Taylor expansion of $f\left(t_{n}+c_{i} k\right)$ has been used as well as changes of subindexes.

As, according to (1),

$$
\begin{aligned}
u\left(t_{n+1}\right) & =u\left(t_{n}\right)+\sum_{m=1}^{p} \frac{k^{m}}{m !} u^{(m)}\left(t_{n}\right) \\
& =u\left(t_{n}\right)+\sum_{m=1}^{p} \frac{k^{m}}{m !}\left[A^{m} u\left(t_{n}\right)+\sum_{r=0}^{m-1} A^{m-r-1} f^{(r)}\left(t_{n}\right)\right]+O\left(k^{p+1}\right),
\end{aligned}
$$

the result follows.

TheOREM 9. If $p=s$ in (4) and (18), for any nodes $\left\{c_{i}\right\}_{i=1}^{s}, \rho_{n}=O\left(k^{s+1}\right)$.

Proof. It suffices to take into account that any polynomial of degree $\leq s-1$ coincides with its interpolant on the nodes $\left\{c_{i}\right\}_{i=1}^{s}$. Therefore, for $r \leq s-1, \sum_{i=1}^{s} c_{i}^{r} l_{i}(\theta)=$ $\theta^{r}$. Using (7), this implies that

$$
\sum_{i=1}^{s} c_{i}^{r} \frac{a_{i, l}}{(l-1) !}=\left\{\begin{array}{lll}
0 & \text { if } & l \neq r+1 \\
1 & \text { if } & l=r+1
\end{array}\right.
$$

or equivalently, for $r \leq s-1$,

$$
\sum_{i=1}^{s} c_{i}^{r} a_{i, r+1}=r !, \quad \sum_{i=1}^{s} c_{i}^{r} a_{i, l}=0, \text { whenever } l \neq r+1 .
$$

Substituting this in the expression for $\rho_{n}$ in Lemma 8 with $p=s$, all the terms in brackets vanish and the result follows.

THEOREM 10. If $p=s+1$ in (4) and (18) and the nodes $\left\{c_{i}\right\}_{i=1}^{s}$ are such that the interpolatory quadrature rule which is based on them is exact for polynomials of degree $\leq s, \rho_{n}=O\left(k^{s+2}\right)$.

Proof. With the same argument as in the previous lemma, all the terms in brackets in the expression of $\rho_{n}$ in Lemma 8 vanish for $m \leq s$. Then, for $m=s+1$, the term in parenthesis vanishes for the same reason when $r \leq s-1$. It just suffices to see what happens when $m=s+1$ and $r=s$. But, as the quadrature rule which is based on $\left\{c_{i}\right\}_{i=1}^{s}$ is assumed to be exact for the polynomial $\theta^{s}$,

$$
\frac{1}{s+1}=\int_{0}^{1} \theta^{s} d \theta=\int_{0}^{1} \sum_{i=1}^{s} c_{i}^{s} l_{i}(\theta) d \theta=\sum_{i=1}^{s} c_{i}^{s} \sum_{l=1}^{s} \frac{a_{i, l}}{l !} .
$$

From this, the result also directly follows. 
We now state the following much more general result:

THEOREM 11. Whenever the nodes $\left\{c_{i}\right\}_{i=1}^{s}$ are such that the interpolatory quadrature rule which is based on them is exact for polynomials of degree $\leq p-1$, considering that value of $p$ in (4) and (18), $\rho_{n}=O\left(k^{p+1}\right)$.

Proof. It suffices to notice that, for $0 \leq r \leq m-1$, with $m \leq p$, due to the hypothesis,

$$
\begin{aligned}
\int_{0}^{1} \int_{0}^{u_{1}} \ldots \int_{0}^{u_{m-r-1}} \theta^{r} d \theta d u_{m-r-1} \ldots d u_{1} \\
\quad=\int_{0}^{1} \int_{0}^{u_{1}} \ldots \int_{0}^{u_{m-r-1}} \sum_{i=1}^{s} c_{i}^{r} l_{i}(\theta) d \theta d u_{m-r-1} \ldots d u_{1} .
\end{aligned}
$$

Now, the left-hand side term above can inductively be proved to be

$$
\frac{1}{r+1} \frac{1}{r+2} \ldots \frac{1}{m}
$$

and the right-hand side can be written as

$$
\begin{aligned}
& \int_{0}^{1} \int_{0}^{u_{1}} \ldots \int_{0}^{u_{m-r-1}} \sum_{i=1}^{s} c_{i}^{r} \sum_{l=1}^{s} a_{i, l} \frac{\theta^{l-1}}{(l-1) !} d \theta d u_{m-r-1} \ldots d u_{1} \\
& =\sum_{l=1}^{s}\left(\sum_{i=1}^{s} c_{i}^{r} a_{i, l}\right) \int_{0}^{1} \int_{0}^{u_{1}} \ldots \int_{0}^{u_{m-r-1}} \frac{\theta^{l-1}}{(l-1) !} d \theta d u_{m-r-1} \ldots d u_{1} \\
& =\sum_{l=1}^{s}\left(\sum_{i=1}^{s} c_{i}^{r} a_{i, l}\right) \frac{1}{(l-1+m-r) !}
\end{aligned}
$$

Then, using Lemma 8, the result directly follows.

From this, the following interesting results are achieved:

COROLlary $12 . \quad$ (i) For the $s$ nodes corresponding to a Gaussian quadrature rule, considering $p=2 s$ in (4) and (18), $\rho_{n}=O\left(k^{2 s+1}\right)$.

(ii) For the $s$ nodes corresponding to a Gaussian-Lobatto quadrature rule, considering $p=2 s-2$ in (4) and (18), $\rho_{n}=O\left(k^{2 s-1}\right)$.

REMARK 13. Due to the fact that the last node of one step is the first of the following, the nodes corresponding to the Gaussian-Lobatto quadrature rule have the advantage that just $s(s-1)$ (instead of $s^{2}$ ) terms of the form $V_{h, n, j, i}$ must be calculated in (27).

4.2. Full discretization error. Let us also consider the error which arises when discretizing (15) and (20) in space.

4.2.1. Local error. To define the local error after full discretization, we consider

$$
\bar{U}_{h, n+1}=\bar{V}_{h, n, 0}(k)+k \sum_{i, j=1}^{s} a_{i, j} \bar{V}_{h, n, j, i}(k),
$$

where

(i) $\bar{V}_{h, n, 0}(\tau)$ is the solution of

$$
\begin{aligned}
\bar{V}_{h, n, 0}^{\prime}(\tau)+L_{h} Q_{h} \partial \hat{v}_{n, 0}^{\prime}(\tau) & =A_{h, 0} \bar{V}_{h, n, 0}(\tau)+A_{h} Q_{h} \partial \hat{v}_{n, 0}(\tau), \\
\bar{V}_{h, n, 0}(0) & =R_{h} u\left(t_{n}\right),
\end{aligned}
$$

with $\hat{v}_{n, 0}(\tau)=\sum_{l=0}^{p} \frac{\tau^{l}}{l !} A^{l} u\left(t_{n}\right)$. 
(ii) $\bar{V}_{h, n, j, i}(\tau)$ is the solution of (22) substituting $P_{h} f\left(t_{n}+c_{i} k\right)$ by $R_{h} f\left(t_{n}+c_{i} k\right)$. More precisely,

$$
\begin{aligned}
\bar{V}_{h, j, n, i}^{\prime}(\tau)= & \left(A_{h, 0}-\frac{j}{\tau} I\right) \bar{V}_{h, j, n, i}(\tau)+A_{h} Q_{h} \partial \hat{v}_{j, n, i}(\tau) \\
& +\frac{1}{(j-1) ! \tau} R_{h} f\left(t_{n}+c_{i} k\right) \\
& +L_{h} Q_{h} \partial\left[\frac{1}{(j-1) ! \tau} f\left(t_{n}+c_{i} k\right)-\frac{j}{\tau} \hat{v}_{j, n, i}(\tau)-\hat{v}_{j, n, i}^{\prime}(\tau)\right], \\
(28) \bar{V}_{h, j, n, i}(0)= & \frac{1}{j !} R_{h} f\left(t_{n}+c_{i} k\right) .
\end{aligned}
$$

Then, we define $\rho_{h, n}=R_{h} u\left(t_{n+1}\right)-\bar{U}_{h, n+1}$ and the following is satisfied.

THEOREM 14. Let us assume that, apart from hypotheses of Section 2, $u$ and $f$ in (1) satisfy

(29) $A^{j} u \in C([0, T], Z), \quad j=0, \ldots, p+1, \quad A^{j} f \in C([0, T], Z), \quad j=0, \ldots, p$.

Then, $\rho_{h, n}=O\left(k \varepsilon_{h}+\left\|\rho_{n}\right\|\right)$, where the constant in Landau notation is independent of $k$ and $h$ and the bounds in Section 4.1 hold for $\rho_{n}$.

Proof. Because of definition,

$$
\begin{aligned}
\rho_{h, n} & =\left(R_{h} u\left(t_{n+1}\right)-R_{h} \bar{u}_{n+1}\right)+\left(R_{h} \bar{u}_{n+1}-\bar{U}_{h, n+1}\right) \\
& =R_{h} \rho_{n}+\left(R_{h} \bar{u}_{n+1}-\bar{U}_{h, n+1}\right),
\end{aligned}
$$

where $\bar{u}_{n}$ and $\rho_{n}$ are those defined in Section 4.1. The fact that (29) is satisfied implies that $\bar{u}_{n+1}$ belongs to $Z$ and therefore $\rho_{n} \in Z$. Moreover, $\left\|\rho_{n}\right\|_{Z}=O\left(\left\|\rho_{n}\right\|\right)$ and, using the same proof as that of Theorem 11 in [4],

$$
R_{h} \rho_{n}=O\left(\left\|\rho_{n}\right\|\right) .
$$

On the other hand,

$(32) R_{h} \bar{u}_{n+1}-\bar{U}_{h, n+1}=R_{h} \bar{v}_{0, n}-\bar{V}_{h, n, 0}(k)+k \sum_{i, j=1}^{s} a_{i, j}\left[R_{h} v_{j, n, i}(k)-\bar{V}_{h, j, n, i}(k)\right]$

where $\bar{v}_{0, n}$ corresponds to (16) with $\alpha=u\left(t_{n}\right)$ and $v_{j, n, i}(\tau)$ corresponds to (17) with $\alpha=f\left(t_{n}+c_{i} k\right)$. In the same way as in the proof of Theorem 4.4 in [4],

$$
R_{h} \bar{v}_{0, n}-\bar{V}_{h, n, 0}(k)=O\left(k \varepsilon_{h}\right) .
$$

Moreover, using Lemma 7,

$$
\begin{aligned}
R_{h} v_{j, n, i}^{\prime}(\tau)= & R_{h} A v_{j, n, i}(\tau)-\frac{j}{\tau} R_{h} v_{j, n, i}(\tau)+\frac{1}{(j-1) ! \tau} R_{h} f\left(t_{n}+c_{i} k\right) \\
= & P_{h} A v_{j, n, i}(\tau)+\left(R_{h}-P_{h}\right) A v_{j, n, i}(\tau)-\frac{j}{\tau} R_{h} v_{j, n, i}(\tau)+\frac{1}{(j-1) ! \tau} R_{h} f\left(t_{n}+c_{i} k\right) \\
= & A_{h, 0} R_{h} v_{j, n, i}(\tau)+A_{h} Q_{h} \partial \hat{v}_{j, n, i}(\tau)-L_{h} Q_{h} \partial A v_{j, n, i}(\tau) \\
& +\left(R_{h}-P_{h}\right) A v_{j, n, i}(\tau)-\frac{j}{\tau} R_{h} v_{j, n, i}(\tau)+\frac{1}{(j-1) ! \tau} R_{h} f\left(t_{n}+c_{i} k\right),
\end{aligned}
$$


and making the difference with (28), it follows that

$$
R_{h} v_{j, n, i}^{\prime}(\tau)-\bar{V}_{h, n, j, i}^{\prime}(\tau)=\left(A_{h, 0}-\frac{j}{\tau} I\right)\left(R_{h} v_{j, n, i}(\tau)-V_{h, n, j, i}(\tau)\right)+\left(R_{h}-P_{h}\right) A v_{j, n, i},
$$

where we have used that

$$
\partial\left[A v_{j, n, i}(\tau)+\frac{1}{(j-1) ! \tau} f\left(t_{n}+c_{i} k\right)-\frac{j}{\tau} \partial \hat{v}_{j, n, i}(\tau)-\partial \hat{v}_{j, n, i}^{\prime}(\tau)\right]=0
$$

because of Lemma 7. Now, due to the same lemma and (28), $R_{h} v_{j, n, i}(0)-\bar{V}_{h, n, j, i}(0)=$ 0 , and therefore

$$
\begin{aligned}
R_{h} v_{j, n, i}(k)-\bar{V}_{h, n, j, i}(k) & =\int_{0}^{k} e^{(k-\tau) A_{h, 0}} \frac{\tau^{j}}{k^{j}}\left(R_{h}-P_{h}\right) A v_{j, n, i}(\tau) d \tau \\
& =k \varphi_{j+1}\left(k A_{h, 0}\right) O\left(\varepsilon_{h}\right)=O\left(k \varepsilon_{h}\right) .
\end{aligned}
$$

Here we have used that $A v_{j, n, i} \in Z$ because of (29) and Lemma 3.3 in [4]. Finally, gathering (30)-(34), the result follows.

4.2.2. Global error. We now study the global error, which we define as $e_{h, n}=$ $P_{h} u\left(t_{n}\right)-U_{h, n}$.

THEOREM 15. Under the same assumptions of Theorem 14,

$$
e_{h, n}=O\left(\frac{1}{k} \max _{0 \leq l \leq n-1}\left\|\rho_{l}\right\|+\varepsilon_{h}\right),
$$

where the constant in Landau notation is independent of $k$ and $h$ and the bounds in Section 4.1 hold for $\rho_{l}$.

Proof. As in the proof of Theorem 4.5 in [4],

$$
\begin{aligned}
e_{h, n+1} & =\left(P_{h} u\left(t_{n+1}\right)-R_{h} u\left(t_{n+1}\right)\right)+R_{h} u\left(t_{n+1}\right)-U_{h, n+1} \\
& =O\left(\varepsilon_{h}\right)+R_{h} u\left(t_{n+1}\right)-U_{h, n+1} .
\end{aligned}
$$

The difference is that now, using (27),

$$
\begin{aligned}
R_{h} u\left(t_{n+1}\right)-U_{h, n+1} & =\rho_{h, n}+\bar{U}_{h, n+1}-U_{h, n+1} \\
& =\rho_{h, n}+\bar{V}_{h, n, 0}(k)-V_{h, n, 0}(k)+k \sum_{i, j=1}^{s} a_{i j}\left(\bar{V}_{h, j, n, i}(k)-V_{h, j, n, i}(k)\right) .
\end{aligned}
$$

As in [4],

$$
\bar{V}_{h, n, 0}-V_{h, n, 0}=e^{k A_{h, 0}}\left(R_{h} u\left(t_{n}\right)-U_{h, n}\right) .
$$

As for $\bar{V}_{h, j, n, i}(k)-V_{h, j, n, i}(k)$, making the difference between (28) and (22),

$\bar{V}_{h, j, n, i}^{\prime}(\tau)-V_{h, j, n, i}^{\prime}(\tau)=\left(A_{h, 0}-\frac{j}{\tau}\right)\left(\bar{V}_{h, j, n, i}(\tau)-V_{h, j, n, i}(\tau)\right)+\frac{1}{(j-1) ! \tau}\left(R_{h}-P_{h}\right) f\left(t_{n}+c_{i} k\right)$,

$\bar{V}_{h, j, n, i}(0)-V_{h, j, n, i}(0)=\frac{1}{j !}\left(R_{h}-P_{h}\right) f\left(t_{n}+c_{i} k\right)$.

Considering then an analogue of Lemma 6 substituting $A_{0}$ by $A_{h, 0}$ and taking into account that $\varphi_{j}(0)=1 / j !(3)$,

$$
\bar{V}_{h, j, n, i}(k)-V_{h, j, n, i}(k)=\varphi_{j}\left(k A_{h, 0}\right)\left(R_{h}-P_{h}\right) f\left(t_{n}+c_{i} k\right)=O\left(\varepsilon_{h}\right) .
$$


Therefore,

$$
R_{h} u\left(t_{n+1}\right)-U_{h, n+1}=e^{k A_{h, 0}}\left(R_{h} u\left(t_{n}\right)-U_{h, n}\right)+\rho_{h, n}+O\left(k \varepsilon_{h}\right) .
$$

This implies that

$$
R_{h} u\left(t_{n+1}\right)-U_{h, n+1}=e^{t_{n+1} A_{h, 0}}\left(R_{h} u(0)-U_{h, 0}\right)+O\left(\frac{1}{k} \max _{0 \leq l \leq n}\left\|\rho_{h, l}\right\|+\varepsilon_{h}\right),
$$

which, together with the first line of (27), (10), (35) and Theorem 14, implies the result.

5. Numerical experiments. In this section we will show some numerical experiments which corroborate the previous results. For that, we have considered parabolic problems with homogeneous and non-homogeneous Dirichlet boundary conditions for which $X=L^{2}(\Omega)$ for a certain spatial domain $\Omega$ and $g \in H^{\frac{1}{2}}(\partial \Omega)$. The fact that these problems can be well fitted under the theory of abstract IBVPs is well justified in $[4,13]$. Moreover, other types of boundary conditions can also be considered although we restrict here to Dirichlet boundary conditions just for the sake of brevity.

As for the space discretization, we have considered here both the standard symmetric 2nd-order finite differences and collocation spectral methods in 1 dimension. For the former, it was already well justified in [4] that the hypotheses which are required on the space discretization are satisfied, at least for the discrete $L^{2}$-norm, $Z=H^{4}(\Omega)$ and $\varepsilon_{h}=O\left(h^{2}\right)$. Besides, a discrete maximum principle (hypothesis (HS)) is well-known to apply [14]. With the collocation spectral methods, those hypotheses are also valid with the discrete $L^{2}$-norm associated to the corresponding GaussianLobatto quadrature rule $\left(\|\cdot\|_{h, G L}\right), Z=H^{m}(\Omega)$ and $\varepsilon_{h}=O\left(J^{2-m}\right)[2,6]$, where $J+1$ is the number of collocation nodes, which is clearly inversely proportional to the diameter space grid $h$. In such a way, the more regular the functions are, the quicker the numerical solution of the elliptic problems converges to the exact solution.

Besides, although in the collocation case the matrix which represents $A_{h, 0}$ is not symmetric any more, Remarks 3 and 5 still apply. Notice that, for every matrix $B$ of dimension $(J-1) \times(J-1)$,

$$
\|B\|_{h, G L}=\left\|D_{J} B D_{J}^{-1}\right\|_{h}
$$

where $D_{J}$ denotes the diagonal matrix which contains the square root of the coefficients of the quadrature rule corresponding to the interior Gauss-Lobatto nodes $\left\{x_{j}\right\}_{j=1}^{J-1}$. (We will denote them by $\left\{\alpha_{j}\right\}_{j=1}^{J-1}$.) Because of this, when $D_{J} B D_{J}^{-1}$ is symmetric, $\|B\|_{h, G L}=\rho(B)$. The fact that $D_{J} A_{h, 0} D_{J}^{-1}$ is symmetric comes from the following: Notice that $\left(A_{h}\right)_{i, j}=L_{j}^{\prime \prime}\left(x_{i}\right)$ where $\left\{L_{j}(x)\right\}$ are the Lagrange polynomials associated to the interior Gauss-Lobatto nodes and those at the boundary. As $\left\{L_{j}(x)\right\}_{j=1}^{J-1}$ vanish at the boundary, integrating by parts, for every $i, j \in\{1, \ldots, J-1\}$,

$$
\int L_{j}^{\prime \prime}(x) L_{i}(x) d x=-\int L_{j}^{\prime}(x) L_{i}^{\prime}(x) d x
$$

As the integrand in the left-hand side is a polynomial of degree $2 J-2$, the corresponding Gaussian-Lobatto quadrature rule integrates it exactly. Therefore,

$$
\alpha_{i} L_{j}^{\prime \prime}\left(x_{i}\right)=-\int L_{j}^{\prime}(x) L_{i}^{\prime}(x) d x=\alpha_{j} L_{i}^{\prime \prime}\left(x_{j}\right),
$$


where, for the last equality, the role of $i$ and $j$ has been interchanged. From this, and using (36) again,

$$
\left\|k A_{h, 0} \sum_{r=1}^{n-1} e^{r k A_{h, 0}}\right\|_{h, G L}=\left\|k D_{J} A_{h, 0} D_{J}^{-1} e^{k(1-\theta) D_{J} A_{h, 0} D_{J}^{-1}}\right\|_{h} .
$$

As $D_{J} A_{h, 0} D_{J}^{-1}$ is symmetric, the matrix inside $\|\cdot\|_{h}$ is also symmetric and therefore

$$
\left\|k A_{h, 0} \sum_{r=1}^{n-1} e^{r k A_{h, 0}}\right\|_{h, G L}=\rho\left(k D_{J} A_{h, 0} D_{J}^{-1} \sum_{r=1}^{n-1} e^{r k D_{J} A_{h, 0} D_{J}^{-1}}\right)=\rho\left(k A_{h, 0} \sum_{r=1}^{n-1} e^{r k A_{h, 0}}\right) .
$$

Secondly, the eigenvalues of $A_{h, 0}$ are negative. This is due to the following: For every polynomial which vanishes at the boundary such that $p(x) \not \equiv 0$,

$$
\int p^{\prime \prime}(x) p(x) d x=-\int\left[p^{\prime}(x)\right]^{2} d x<0 .
$$

Considering $p(x)=\sum_{i=1}^{J-1} \beta_{i} L_{i}(x)$ and using the Gauss-Lobatto quadrature rule and the definition of Lagrange polynomials,

$$
\sum_{k=1}^{J-1} \alpha_{k}\left(\sum_{i=1}^{J-1} \beta_{i} L_{i}^{\prime \prime}\left(x_{k}\right)\right)\left(\sum_{j=1}^{J-1} \beta_{j} L_{j}\left(x_{k}\right)\right)=\sum_{i, k=1}^{J-1} \alpha_{k} \beta_{i} \beta_{k} L_{i}^{\prime \prime}\left(x_{k}\right)<0 .
$$

This can be rewritten as $\vec{\beta}^{T} D_{J}^{2} A_{h, 0} \vec{\beta}<0$ for every vector $\vec{\beta} \neq \overrightarrow{0}$, or equivalently, $\left(D_{J} \vec{\beta}\right)^{T} D_{J} A_{h, 0} D_{J}^{-1}\left(D_{J} \vec{\beta}\right)<0$, which implies that $D_{J} A_{h, 0} D_{J}^{-1}$ has negative eigenvalues and so has $A_{h, 0}$.

For both types of discretizations which have been considered here, $L_{h} Q_{h} \partial \equiv 0$ and therefore formulas (21) and (26) simplify a little bit. However, other possible discretizations (as those considered in [4]) are also possible, for which that simplification cannot be made.

In all cases, we have considered the one-dimensional problem

$$
\begin{aligned}
u_{t}(x, t) & =u_{x x}(x, t)+f(x, t), \quad 0 \leq t \leq 1, \quad 0 \leq x \leq 1, \\
u(x, 0) & =u_{0}(x), \\
u(0, t) & =g_{0}(t), \quad u(1, t)=g_{1}(t),
\end{aligned}
$$

with the corresponding functions $f, u_{0}, g_{0}$ and $g_{1}$ which make that $u(x, t)=x(1-$ $x) e^{-t}$ or $u(x, t)=e^{x-t}$ are solutions of the problem. These functions satisfy regularity hypotheses (4) and (29) for any natural number $p$.

5.1. Trapezoidal rule. We begin by considering the trapezoidal rule in time and the second-order finite differences in space. We have considered $h=10^{-3}$ so that the error in space is negligible. The trapezoidal rule corresponds to $s=2$ but is just exact for polynomials of degree $\leq 1$. Therefore, one of the hypothesis of Theorem 2 is not satisfied and we can just apply Theorem 1 when discretizating firstly in space and then in time with the solution which satisfies $g_{0}(t)=g_{1}(t)=0$. That theorem states that, with respect to the time stepsize $k$, the local and global error should show orders 3 and 2 respectively and we can check that really happens in Table 1. For the same problem, but applying the technique which is suggested in this paper (27) with $p=2$, Theorems 9, 14 and 15 state that also the local and global error should show orders 3 


\begin{tabular}{|c|c|c|c|c|c|c|c|c|}
\hline & \multicolumn{4}{|c|}{ Classical approach } & \multicolumn{4}{c|}{ Suggested approach } \\
\hline $\mathrm{k}$ & Loc. err. & ord. & Glob. err. & ord. & Loc. err. & ord. & Glob. err. & ord. \\
\hline $1 / 10$ & $8.0170 \mathrm{e}-5$ & & $5.5395 \mathrm{e}-5$ & & $1.5334 \mathrm{e}-4$ & & $9.8091 \mathrm{e}-5$ & \\
\hline $1 / 20$ & $1.2961 \mathrm{e}-5$ & 2.6 & $1.3953 \mathrm{e}-5$ & 2.0 & $1.9139 \mathrm{e}-5$ & 3.0 & $1.8575 \mathrm{e}-5$ & 2.4 \\
\hline $1 / 40$ & $1.8644 \mathrm{e}-6$ & 2.8 & $3.4952 \mathrm{e}-6$ & 2.0 & $2.3902 \mathrm{e}-6$ & 3.0 & $4.0262 \mathrm{e}-6$ & 2.2 \\
\hline $1 / 80$ & $2.5316 \mathrm{e}-7$ & 2.9 & $8.7426 \mathrm{e}-7$ & 2.0 & $2.9862 \mathrm{e}-7$ & 3.0 & $9.3773 \mathrm{e}-7$ & 2.1 \\
\hline $1 / 160$ & $3.3354 \mathrm{e}-8$ & 2.9 & $2.1860 \mathrm{e}-7$ & 2.0 & $3.7318 \mathrm{e}-8$ & 3.0 & $2.2635 \mathrm{e}-7$ & 2.0 \\
\hline $1 / 320$ & $4.3171 \mathrm{e}-9$ & 3.0 & $5.4651 \mathrm{e}-8$ & 2.0 & $4.6641 \mathrm{e}-9$ & 3.0 & $5.5610 \mathrm{e}-8$ & 2.0 \\
\hline
\end{tabular}

TABLE 1

Trapezoidal rule, $h=10^{-3}, u(x, t)=x(1-x) e^{-t}$

\begin{tabular}{|c|c|c|c|c|c|c|c|c|}
\hline & \multicolumn{4}{|c|}{ Classical approach } & \multicolumn{4}{c|}{ Suggested approach } \\
\hline $\mathrm{k}$ & Loc. err. & ord. & Glob. err. & ord. & Loc. err. & ord. & Glob. err. & ord. \\
\hline $1 / 10$ & $7.4531 \mathrm{e}-4$ & & $4.8108 \mathrm{e}-4$ & & $2.0144 \mathrm{e}-4$ & & $1.3742 \mathrm{e}-4$ & \\
\hline $1 / 20$ & $1.5446 \mathrm{e}-4$ & 2.3 & $1.2476 \mathrm{e}-4$ & 2.0 & $2.8775 \mathrm{e}-5$ & 2.8 & $3.0165 \mathrm{e}-5$ & 2.2 \\
\hline $1 / 40$ & $3.3863 \mathrm{e}-5$ & 2.2 & $3.2074 \mathrm{e}-5$ & 2.0 & $3.9084 \mathrm{e}-6$ & 2.9 & $7.0453 \mathrm{e}-6$ & 2.1 \\
\hline $1 / 80$ & $7.3906 \mathrm{e}-6$ & 2.2 & $8.1809 \mathrm{e}-6$ & 2.0 & $5.1475 \mathrm{e}-7$ & 2.9 & $1.6979 \mathrm{e}-6$ & 2.0 \\
\hline $1 / 160$ & $1.5848 \mathrm{e}-6$ & 2.2 & $2.0770 \mathrm{e}-6$ & 2.0 & $6.6122 \mathrm{e}-8$ & 3.0 & $4.1324 \mathrm{e}-7$ & 2.0 \\
\hline $1 / 320$ & $3.3666 \mathrm{e}-7$ & 2.2 & $5.2777 \mathrm{e}-7$ & 2.0 & $8.1531 \mathrm{e}-9$ & 3.0 & $9.8459 \mathrm{e}-8$ & 2.1 \\
\hline
\end{tabular}

TABLE 2

Trapezoidal rule, $h=10^{-3}, u(x, t)=e^{x-t}$

and 2 respectively and that is what we can in fact observe in the same table. We can see that, although the local order is a bit more clear with the suggested technique, the size of the errors is slightly bigger with the suggested approach. Therefore, it seems that, in this particular problem, the error constants are bigger with the suggested technique and it is not worth the additional cost of calculating terms which contain $\varphi_{3}\left(k A_{h, 0}\right)$ and $\varphi_{4}\left(k A_{h, 0}\right)$.

The comparison is more advantageous for the suggested technique when the solution is such that it does not vanish at the boundary. Then, Theorem 4 and Remark 5 state that the local and global error should show order 2 with the classical approach and that can be checked in Table 2. However, with the suggested strategy, as with the vanishing boundary conditions case, the theorems in this paper prove local order 3 and global order 2, which can again be checked in the same table. The fact that we manage to increase the order in the local error makes that the global errors, although always of order 2, are smaller with the suggested technique than with the classical approach. Nevertheless, the comparison between both techniques will be more beneficial for the technique which is suggested in the paper when the classical (non-exponential) order of the quadrature rule increases.

5.2. Simpson rule. In this subsection we consider Simpson rule in time and a collocation spectral method in space with 40 nodes so that the error in space is negligible. As Simpson rule corresponds to $s=3$ and the interpolatory quadrature rule which is based in those 3 nodes is exact for polynomials of degree $\leq 3$, we can take $p=4$ in Theorem 10 and achieve orders 5 and 4 for the local and global error respectively with the technique suggested here. However, with the classical approach, at least in the common case that $g(t) \not \equiv 0$, Theorem 4 and Remark 5 give just order 3 for the local and global error. These results can be checked in Table 3. Moreover, the 


\begin{tabular}{|c|c|c|c|c|c|c|c|c|}
\hline & \multicolumn{4}{|c|}{ Classical approach } & \multicolumn{4}{c|}{ Suggested approach } \\
\hline $\mathrm{k}$ & Loc. err. & ord. & Glob. err. & ord. & Loc. err. & ord. & Glob. err. & ord. \\
\hline $1 / 2$ & $8.0718 \mathrm{e}-4$ & & $4.9507 \mathrm{e}-4$ & & $2.7496 \mathrm{e}-4$ & & $1.6821 \mathrm{e}-4$ & \\
\hline $1 / 4$ & $8.3265 \mathrm{e}-5$ & 3.3 & $4.2862 \mathrm{e}-5$ & 3.5 & $8.5778 \mathrm{e}-6$ & 5.0 & $4.4156 \mathrm{e}-6$ & 5.2 \\
\hline $1 / 8$ & $8.6214 \mathrm{e}-6$ & 3.3 & $4.0561 \mathrm{e}-6$ & 3.4 & $2.6785 \mathrm{e}-7$ & 5.0 & $1.5345 \mathrm{e}-7$ & 4.8 \\
\hline $1 / 16$ & $1.0500 \mathrm{e}-6$ & 3.0 & $4.4103 \mathrm{e}-7$ & 3.2 & $8.3681 \mathrm{e}-9$ & 5.0 & $6.7803 \mathrm{e}-9$ & 4.5 \\
\hline $1 / 32$ & $1.1622 \mathrm{e}-7$ & 3.2 & $4.6864 \mathrm{e}-8$ & 3.2 & $2.6148 \mathrm{e}-10$ & 5.0 & $3.5342 \mathrm{e}-10$ & 4.3 \\
\hline $1 / 64$ & $1.2378 \mathrm{e}-8$ & 3.2 & $4.9423 \mathrm{e}-9$ & 3.2 & $8.1711 \mathrm{e}-12$ & 5.0 & $2.0189 \mathrm{e}-11$ & 4.1 \\
\hline
\end{tabular}

TABLE 3

Simpson's rule, $J=61, u(x, t)=e^{x-t}$

\begin{tabular}{|c|c|c|c|c|c|c|c|c|}
\hline & \multicolumn{4}{|c|}{ Classical approach } & \multicolumn{4}{c|}{ Suggested approach } \\
\hline $\mathrm{k}$ & Loc. err. & ord. & Glob. err. & ord. & Loc. err. & ord. & Glob. err. & ord. \\
\hline $1 / 4$ & $8.2639 \mathrm{e}-3$ & & $4.3743 \mathrm{e}-3$ & & $4.9483 \mathrm{e}-3$ & & $2.5685 \mathrm{e}-3$ & \\
\hline $1 / 8$ & $1.8874 \mathrm{e}-3$ & 2.1 & $1.1548 \mathrm{e}-3$ & 1.9 & $6.4427 \mathrm{e}-4$ & 2.9 & $3.7820 \mathrm{e}-4$ & 2.8 \\
\hline $1 / 16$ & $3.6716 \mathrm{e}-4$ & 2.4 & $2.9791 \mathrm{e}-4$ & 2.0 & $8.2199 \mathrm{e}-5$ & 3.0 & $6.9202 \mathrm{e}-5$ & 2.4 \\
\hline $1 / 32$ & $7.6916 \mathrm{e}-5$ & 2.2 & $7.6389 \mathrm{e}-5$ & 2.0 & $1.0381 \mathrm{e}-5$ & 3.0 & $1.4677 \mathrm{e}-5$ & 2.2 \\
\hline $1 / 64$ & $1.6803 \mathrm{e}-5$ & 2.2 & $1.9445 \mathrm{e}-5$ & 2.0 & $1.3043 \mathrm{e}-6$ & 3.0 & $3.3796 \mathrm{e}-6$ & 2.1 \\
\hline $1 / 128$ & $3.6111 \mathrm{e}-6$ & 2.2 & $4.9232 \mathrm{e}-6$ & 2.0 & $1.6345 \mathrm{e}-7$ & 3.0 & $8.1115 \mathrm{e}-7$ & 2.1 \\
\hline
\end{tabular}

TABLE 4

Midpoint rule, $J=61, u(x, t)=x(1-x) e^{-t}$

size of the global error, even for the bigger timestepsizes is smaller with the suggested technique.

We also want to remark here that the trapezoidal and Simpson rules correspond to Gauss-Lobatto quadrature rules with $s=2$ and $s=3$ respectively and therefore Corollary 12 (ii) and Remark 13 apply.

5.3. Gaussian rules. In order to achieve the highest accuracy given a certain number of nodes, we consider in this subsection Gaussian quadrature rules. More precisely, those corresponding to $s=1,2,3,4$. As space discretization, we have considered again the same spectral collocation method of the previous subsection. Following Corollary 12 (i) and Theorem 14, even for non-vanishing boundary conditions, taking $p=2 s$ in (21) and (26) the local error in time should show order $2 s+1$ and the global error, using Theorem 15, order $2 s$. This should be compared with the order $s+1$ which is proved for the classical approach when $g(t) \equiv 0$ in Theorem 2 and the order $s$ for the local and global error when $g(t) \not \equiv 0$, which comes from Theorem 4 and Remark 5. In Tables 4 and 5 we see the results which correspond to $s=1$ and $s=2$ respectively for the vanishing boundary conditions case. Although for $s=1$ there is not an improvement on the global order for the suggested technique, the errors are a bit smaller. Of course the benefits are more evident with $s=2$. For the non-vanishing boundary conditions case, Tables $6,7,8$ and 9 show the results which correspond to $s=1,2,3$ and 4 respectively. When avoiding order reduction, the results are much better than with the classical approach. Not only the order is bigger but also the size of the errors is smaller from the very beginning. We notice that the global order is even a bit better than expected for the first values of $k$.

Finally, although it is not an aim of this paper, in order to compare roughly the 


\begin{tabular}{|c|c|c|c|c|c|c|c|c|}
\hline & \multicolumn{4}{|c|}{ Classical approach } & \multicolumn{4}{c|}{ Suggested approach } \\
\hline $\mathrm{k}$ & Loc. err. & ord. & Glob. err. & ord. & Loc. err. & ord. & Glob. err. & ord. \\
\hline $1 / 2$ & $8.9292 \mathrm{e}-4$ & & $5.4788 \mathrm{e}-4$ & & $8.2591 \mathrm{e}-5$ & & $5.0573 \mathrm{e}-5$ & \\
\hline $1 / 4$ & $8.6746 \mathrm{e}-5$ & 3.4 & $4.5296 \mathrm{e}-5$ & 3.6 & $2.8465 \mathrm{e}-6$ & 4.9 & $1.4782 \mathrm{e}-6$ & 5.1 \\
\hline $1 / 8$ & $8.1186 \mathrm{e}-6$ & 3.4 & $3.9933 \mathrm{e}-6$ & 3.5 & $9.3457 \mathrm{e}-8$ & 4.9 & $5.4928 \mathrm{e}-8$ & 4.7 \\
\hline $1 / 16$ & $1.0237 \mathrm{e}-6$ & 3.0 & $4.3449 \mathrm{e}-7$ & 3.2 & $2.9938 \mathrm{e}-9$ & 5.0 & $2.5254 \mathrm{e}-9$ & 4.4 \\
\hline $1 / 32$ & $1.1415 \mathrm{e}-7$ & 3.2 & $4.6048 \mathrm{e}-8$ & 3.2 & $9.4726 \mathrm{e}-11$ & 5.0 & $1.3424 \mathrm{e}-10$ & 4.2 \\
\hline $1 / 64$ & $1.2112 \mathrm{e}-8$ & 3.2 & $4.8418 \mathrm{e}-9$ & 3.2 & $2.9786 \mathrm{e}-12$ & 5.0 & $7.7190 \mathrm{e}-12$ & 4.1 \\
\hline
\end{tabular}

TABLE 5

Gaussian rule with $s=2, J=61, u(x, t)=x(1-x) e^{-t}$

\begin{tabular}{|c|c|c|c|c|c|c|c|c|}
\hline & \multicolumn{4}{|c|}{ Classical approach } & \multicolumn{4}{c|}{ Suggested approach } \\
\hline $\mathrm{k}$ & Loc. err. & ord. & Glob. err. & ord. & Loc. err. & ord. & Glob. err. & ord. \\
\hline $1 / 8$ & $6.6985 \mathrm{e}-2$ & & $2.8814 \mathrm{e}-2$ & & $1.5650 \mathrm{e}-3$ & & $8.6254 \mathrm{e}-4$ & \\
\hline $1 / 16$ & $3.0444 \mathrm{e}-2$ & 1.1 & $1.2167 \mathrm{e}-2$ & 1.2 & $1.8673 \mathrm{e}-4$ & 3.1 & $1.4048 \mathrm{e}-4$ & 2.6 \\
\hline $1 / 32$ & $1.3218 \mathrm{e}-2$ & 1.2 & $5.1128 \mathrm{e}-3$ & 1.2 & $2.2356 \mathrm{e}-5$ & 3.1 & $2.7661 \mathrm{e}-5$ & 2.3 \\
\hline $1 / 64$ & $5.6269 \mathrm{e}-3$ & 1.2 & $2.1472 \mathrm{e}-3$ & 1.2 & $2.6947 \mathrm{e}-6$ & 3.1 & $6.1319 \mathrm{e}-6$ & 2.2 \\
\hline $1 / 128$ & $2.3791 \mathrm{e}-3$ & 1.2 & $9.0138 \mathrm{e}-4$ & 1.2 & $3.2718 \mathrm{e}-7$ & 3.0 & $1.4450 \mathrm{e}-6$ & 2.1 \\
\hline $1 / 256$ & $1.0015 \mathrm{e}-3$ & 1.2 & $3.7813 \mathrm{e}-4$ & 1.2 & $3.9993 \mathrm{e}-8$ & 3.0 & $3.5085 \mathrm{e}-7$ & 2.0 \\
\hline \multicolumn{8}{|c|}{ TABLe 6} \\
\hline
\end{tabular}

results in terms of computational cost, let us concentrate on Gaussian quadrature rules of the same order $2 s$ when integrating a non-vanishing boundary value problem. When considering $2 s$ nodes with the classical approach, $2 s$ evaluations of the source term $f$ must be made at each step and the $2 s$ operators $\left\{\varphi_{j}\left(k A_{h, 0}\right)\right\}_{j=1}^{2 s}$ are needed, which will be multiplied by vectors with all its components varying in principle at each step. However, with the suggested technique and $s$ nodes, just $s$ evaluations of the source term $f$ must be made although $3 s$ operators $\left\{\varphi_{j}\left(k A_{h, 0}\right)\right\}_{j=1}^{3 s}$ are needed. Nevertheless, from these $3 s$, just the first $s$ of them are multiplied by vectors which change independently in all their components at each step. The other $2 s$ are multiplied by vectors which just contain information on the boundary. Therefore, with finite differences many components vanish and, with Gauss-Lobatto spectral methods, those vectors are just a time-dependent linear combination of two vectors which do not change with time. With Gauss-Lobatto methods, as $A_{h, 0}$ is not sparse but its size is moderate, we have calculated once and for all at the very beginning $e^{k A_{h, 0}}, \varphi_{j}\left(k A_{h, 0}\right)$, $j=1, \ldots, s$ and the two necessary vectors derived from $\varphi_{j}\left(k A_{h, 0}\right), j=s+1, \ldots, 3 s$. Then, in (27) the terms containing the former at each step require $O\left(J^{2}\right)$ operations while the terms containing the latter just require $O(J)$ operations. With finite differences, as the matrix $A_{h, 0}$ is sparse and usually bigger, we have applied general Krylov subroutines [11] to calculate all the required terms at each step.

We offer a particular comparison for order 2 with Gauss-Lobatto spectral space discretization on the one hand and 2nd-order finite differences on the other, and considering the implementation described above in each case. In Figure 1 we can see that, for the former, the suggested technique is more than twice cheaper than the classical one and, with the latter in Figure 2, the comparison is not so advantageous for the suggested technique but it is still cheaper than the classical approach. We also 


\begin{tabular}{|c|c|c|c|c|c|c|c|c|}
\hline & \multicolumn{4}{|c|}{ Classical approach } & \multicolumn{4}{c|}{ Suggested approach } \\
\hline $\mathrm{k}$ & Loc. err. & ord. & Glob. err. & ord. & Loc. err. & ord. & Glob. err. & ord. \\
\hline $1 / 2$ & $1.9328 \mathrm{e}-2$ & & $1.1743 \mathrm{e}-2$ & & $7.9408 \mathrm{e}-4$ & & $4.8503 \mathrm{e}-4$ & \\
\hline $1 / 4$ & $4.8715 \mathrm{e}-3$ & 2.0 & $2.3068 \mathrm{e}-3$ & 2.3 & $2.2565 \mathrm{e}-5$ & 5.1 & $1.1356 \mathrm{e}-5$ & 5.4 \\
\hline $1 / 8$ & $1.1460 \mathrm{e}-3$ & 2.1 & $4.7811 \mathrm{e}-4$ & 2.3 & $6.3799 \mathrm{e}-7$ & 5.1 & $3.3399 \mathrm{e}-7$ & 5.1 \\
\hline $1 / 16$ & $2.5199 \mathrm{e}-4$ & 2.2 & $9.8750 \mathrm{e}-5$ & 2.3 & $1.8167 \mathrm{e}-8$ & 5.1 & $1.2423 \mathrm{ee}-8$ & 4.7 \\
\hline $1 / 32$ & $5.4061 \mathrm{e}-5$ & 2.2 & $2.0543 \mathrm{e}-5$ & 2.3 & $5.2222 \mathrm{e}-10$ & 5.1 & $5.7608 \mathrm{e}-10$ & 4.4 \\
\hline $1 / 64$ & $1.1476 \mathrm{e}-5$ & 2.2 & $4.2930 \mathrm{e}-6$ & 2.3 & $1.4918 \mathrm{e}-11$ & 5.1 & $2.9580 \mathrm{e}-11$ & 4.3 \\
\hline
\end{tabular}

TABLE 7

Gaussian rule with $s=2, J=61, u(x, t)=e^{x-t}$

\begin{tabular}{|c|c|c|c|c|c|c|c|c|}
\hline & \multicolumn{4}{|c|}{ Classical approach } & \multicolumn{4}{c|}{ Suggested approach } \\
\hline $\mathrm{k}$ & Loc. err. & ord. & Glob. err. & ord. & Loc. err. & ord. & Glob. err. & ord. \\
\hline $1 / 2$ & $8.4732 \mathrm{e}-4$ & & $5.1405 \mathrm{e}-4$ & & $3.0107 \mathrm{e}-6$ & & $1.8363 \mathrm{e}-6$ & \\
\hline $1 / 4$ & $1.0734 \mathrm{e}-4$ & 3.0 & $5.0710 \mathrm{e}-5$ & 3.3 & $2.0423 \mathrm{e}-8$ & 7.2 & $1.0082 \mathrm{e}-8$ & 7.5 \\
\hline $1 / 8$ & $1.2260 \mathrm{e}-5$ & 3.1 & $5.1107 \mathrm{e}-6$ & 3.3 & $1.3840 \mathrm{e}-10$ & 7.2 & $6.6752 \mathrm{e}-11$ & 7.2 \\
\hline $1 / 16$ & $1.3379 \mathrm{e}-6$ & 3.2 & $5.2398 \mathrm{e}-7$ & 3.3 & $9.4087 \mathrm{e}-13$ & 7.2 & $5.3198 \mathrm{e}-13$ & 7.0 \\
\hline $1 / 32$ & $1.4335 \mathrm{e}-7$ & 3.2 & $5.4413 \mathrm{e}-8$ & 3.3 & $6.4275 \mathrm{e}-15$ & 7.2 & $5.3534 \mathrm{e}-15$ & 6.6 \\
\hline $1 / 64$ & $1.5182 \mathrm{e}-8$ & 3.2 & $5.6735 \mathrm{e}-9$ & 3.3 & $4.4259 \mathrm{e}-17$ & 7.2 & $6.6517 \mathrm{e}-17$ & 6.3 \\
\hline
\end{tabular}

TABLE 8

Gaussian rule with $s=3, J=61, u(x, t)=e^{x-t}$

remark that, in any case, the more expensive the source function $f$ is to evaluate, the more advantageous the suggested technique with $s$ nodes will be against the classical approach with $2 s$ nodes.

Moreover, in the same figures, we also compare with the Lawson midpoint rule avoiding order reduction according to [4], which is described in this case by

$$
\begin{gathered}
U_{h, n+1}=V_{h, n, 0}(k)+k\left[e^{\frac{k}{2} A_{h, 0}} P_{h} f\left(t_{n}+\frac{k}{2}\right)+\frac{k}{2} \varphi_{1}\left(\frac{k}{2} A_{h, 0}\right) A_{h} Q_{h} \partial f\left(t_{n}+\frac{k}{2}\right)\right. \\
\left.+\frac{1}{4} k^{2} \varphi_{2}\left(\frac{k}{2} A_{h, 0}\right) A_{h} Q_{h} \partial A f\left(t_{n}+\frac{k}{2}\right)\right],
\end{gathered}
$$

with $V_{h, n, 0}$ that in (21) with $p=2$, i.e.,

$$
\begin{aligned}
V_{h, n, 0}= & e^{k A_{h, 0}} U_{h, n}+k \varphi_{1}\left(k A_{h, 0}\right) A_{h} Q_{h} \partial u\left(t_{n}\right)+k^{2} \varphi_{2}\left(k A_{h, 0}\right) A_{h} Q_{h} \partial A u\left(t_{n}\right) \\
& +k^{3} \varphi_{3}\left(k A_{h, 0}\right) A_{h} Q_{h} \partial A^{2} u\left(t_{n}\right) .
\end{aligned}
$$

If we compare with the exponential midpoint rule which is suggested in this paper, which is given by

$$
\begin{gathered}
U_{h, n+1}=V_{h, n, 0}(k)+k\left[\varphi_{1}\left(k A_{h, 0}\right) P_{h} f\left(t_{n}+\frac{k}{2}\right)+k \varphi_{2}\left(k A_{h, 0}\right) A_{h} Q_{h} \partial f\left(t_{n}+\frac{k}{2}\right)\right. \\
\left.+k^{2} \varphi_{3}\left(k A_{h, 0}\right) A_{h} Q_{h} \partial A f\left(t_{n}+\frac{k}{2}\right)\right]
\end{gathered}
$$

we can see that now there are no terms in $e^{\frac{k}{2} A_{h, 0}}, \varphi_{1}\left(\frac{k}{2} A_{h, 0}\right), \varphi_{2}\left(\frac{k}{2} A_{h, 0}\right)$ and moreover, we can group together the terms in $\varphi_{1}\left(k A_{h, 0}\right), \varphi_{2}\left(k A_{h, 0}\right)$ and $\varphi_{3}\left(k A_{h, 0}\right)$. 


\begin{tabular}{|c|c|c|c|c|c|c|c|c|}
\hline & \multicolumn{4}{|c|}{ Classical approach } & \multicolumn{4}{c|}{ Suggested approach } \\
\hline $\mathrm{k}$ & Loc. err. & ord. & Glob. err. & ord. & Loc. err. & ord. & Glob. err. & ord. \\
\hline $1 / 2$ & $2.7746 \mathrm{e}-5$ & & $1.6829 \mathrm{e}-5$ & & $8.1253 \mathrm{e}-9$ & & $4.9495 \mathrm{e}-9$ & \\
\hline $1 / 4$ & $1.7137 \mathrm{e}-6$ & 4.0 & $8.0951 \mathrm{e}-7$ & 4.4 & $1.3502 \mathrm{e}-11$ & 9.2 & $6.5604 \mathrm{e}-12$ & 9.6 \\
\hline $1 / 8$ & $9.6826 \mathrm{e}-8$ & 4.1 & $4.0363 \mathrm{e}-8$ & 4.3 & $2.2443 \mathrm{e}-14$ & 9.2 & $1.0107 \mathrm{e}-14$ & 9.3 \\
\hline $1 / 16$ & $5.2833 \mathrm{e}-9$ & 4.2 & $2.0690 \mathrm{e}-9$ & 4.3 & $3.7267 \mathrm{e}-17$ & 9.2 & $1.7380 \mathrm{e}-17$ & 9.2 \\
\hline $1 / 32$ & $2.8240 \mathrm{e}-10$ & 4.2 & $1.0719 \mathrm{e}-10$ & 4.3 & $6.2266 \mathrm{e}-20$ & 9.2 & $3.5644 \mathrm{e}-20$ & 8.9 \\
\hline
\end{tabular}

TABLE 9

Gaussian rule with $s=4, J=61, u(x, t)=e^{x-t}$

When using the implementation which is described above for Gauss-Lobatto spectral space discretization, those matrices are stored at the very beginning and the advantage of the rule suggested here above Lawson one is not so big. Notice that, with Lawson rule, 2 expensive applications of matrices over vectors are performed (those corresponding to $e^{k A_{h, 0}}$ and $e^{\frac{k}{2} A_{h, 0}}$ ) while the other 5 ones corresponding to $\varphi_{1}\left(k A_{h, 0}\right), \varphi_{2}\left(k A_{h, 0}\right), \varphi_{3}\left(k A_{h, 0}\right), \varphi_{1}\left(\frac{k}{2} A_{h, 0}\right)$ and $\varphi_{2}\left(\frac{k}{2} A_{h, 0}\right)$ are much cheaper because they just act over the boundaries. Meanwhile, with the rule suggested here, again 2 of them are expensive (those corresponding to $e^{k A_{h, 0}}$ and $\varphi_{1}\left(k A_{h, 0}\right)$ ) and there are just other 2 (corresponding to $\varphi_{2}\left(k A_{h, 0}\right)$ and $\varphi_{3}\left(k A_{h, 0}\right)$ ), which are cheap. Because of this, the rule which is studied in this paper outperforms Lawson one but the difference is not so big. (Look at Figure 1.)

However, for more general problems in which the calculation or the storage of the big matrices is not possible, the advantage of the rule studied here above Lawson is much more pronounced, as it corresponds to calculating additionally through Krylov methods the terms in $e^{\frac{k}{2} A_{h, 0}}, \varphi_{1}\left(\frac{k}{2} A_{h, 0}\right), \varphi_{2}\left(\frac{k}{2} A_{h, 0}\right)$, which require approximately the same cost as those in $e^{k A_{h, 0}}, \varphi_{1}\left(k A_{h, 0}\right), \varphi_{2}\left(k A_{h, 0}\right)$ and $\varphi_{3}\left(k A_{h, 0}\right)$ and which are needed with both methods [11]. That difference is clearly observed in Figure 2.

Besides, although not explicitly done here for the sake of brevity, with the same argument it can be deduced that the bigger $s$ is, the bigger the advantage of the rules suggested here above Lawson ones.

\section{REFERENCES}

[1] I. Alonso-Mallo, Rational methods with optimal order of convergence for partial differential equations, Appl. Num. Math. 35 (2000), 265-292.

[2] I. Alonso-Mallo, B. Cano and J. C. Jorge, Spectral-Fractional Step Runge-Kutta Discretizations for Initial Boundary Value Problems with Time-Dependent Boundary Conditions, Math. Comput. 73 (2004), 1801-1825.

[3] I. Alonso-Mallo, B. Cano and N. Reguera, Analysis of order reduction when integrating linear initial boundary value problems with Lawson methods, Appl. Numer. Math., 118 (2017) $64-74$.

[4] I. Alonso-Mallo, B. Cano and N. Reguera, Avoiding order reduction when integrating linear initial boundary value problems with Lawson methods, IMA J. Num. Anal., 37 (2017), 20912119.

[5] I. Alonso-Mallo, B. Cano and N. Reguera, Avoiding order reduction when integrating reaction-diffusion boundary value problems with exponential splitting methods, arXiv:1705.01857.

[6] C. Bernardy And Y. Maday, Approximations spectrales de problemes aux limites elliptiques, Springer-Verlag France, Paris, 1992.

[7] T. GöCKLer AND V. GRImm, Convergence analysis of an extended Krylov subspace method for the approximation of operator functions in exponential integrators, SIAM J. Numer. Anal. 51 (4) (2013), 2189-2213 


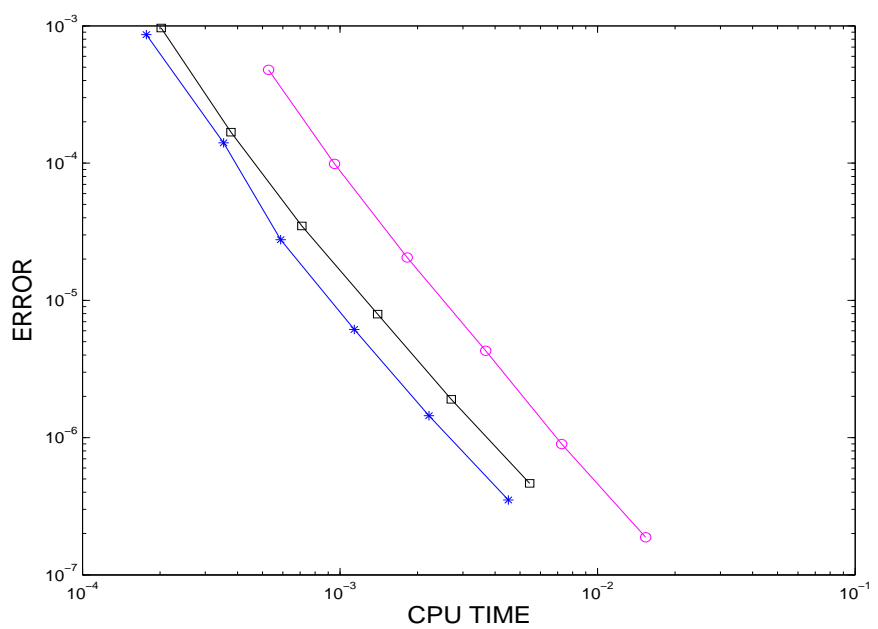

FIG. 1. Error against CPU time when integrating problem (37) with exact solution $u(x, t)=$ $e^{x-t}$, using Gauss-Lobatto spectral method in space and, in time, the classical approach of Gaussian rule with $s=2$ (pink circles), the suggested technique for midpoint rule (blue asterisks) and Lawson midpoint rule without order reduction (black squares)

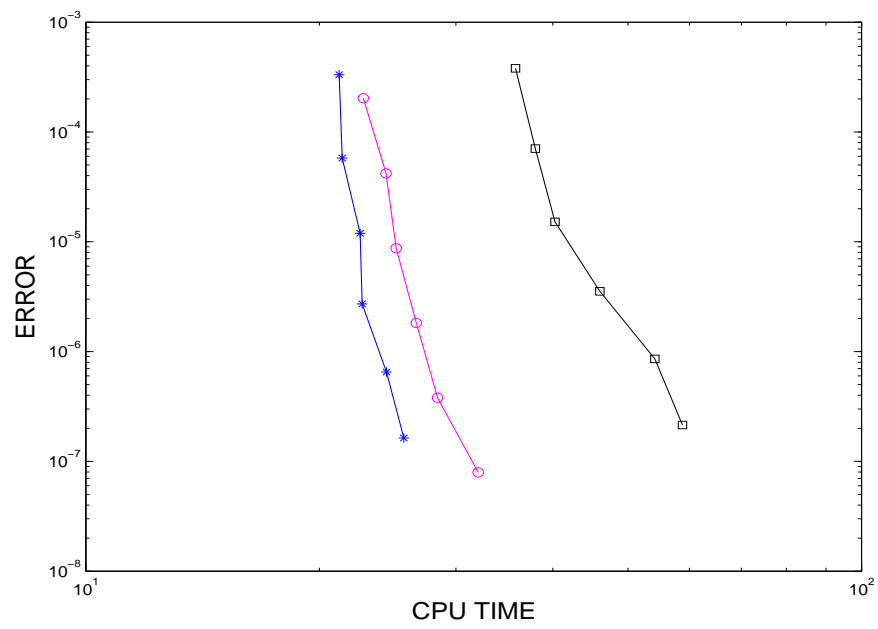

FIG. 2. Error against $C P U$ time when integrating problem (37) with exact solution $u(x, t)=$ $e^{x-t}$, using second-order finite differences in space and, in time, the classical approach of Gaussian rule with $s=2$ (pink circles), the suggested technique for midpoint rule (blue asterisks) and Lawson midpoint rule without order reduction (black squares)

[8] M. Hochbruck and A. Ostermann, Exponential Runge-Kutta methods for parabolic problems, Appl. Numer. Math. 53 (2005), no. 2-4, 323-339.

[9] M. Hochbruck and A. Ostermann, Exponential integrators, Acta Numerica (2010), 209-286.

[10] J. D. LaWson, Generalized Runge-Kutta processes for stable systems with large Lipschitz constants, SIAM J. Numer. Anal. 4 (1967) 372-380.

[11] J. Niesen, AND W. M. Wright, Algorithm 919: a Krylov subspace algorithm for evaluating the $\varphi$-functions appearing in exponential integrators, ACM Trans. Math. Software 38, no. 3, Art. 22 (2012).

[12] C. Palencia and I. Alonso-Mallo, Abstract initial boundary value problems, Proc. Royal Soc. Edinburgh 124A (1994), 879-908. 
617 [13] A. Quarteroni And A. Valli, Numerical Approximation of Partial Differential Equations, $618 \quad$ Springer-verlag, Berlin, 1994.

619 [14] J. C. Strikwerda, Finite Difference Schemes and Partial Differential Equations, Wadsworth 620 \& Brooks, United States of America, 1989. 